\title{
Combined inclusive diffractive cross sections measured with forward proton spectrometers in deep inelastic ep scattering at HERA
}

\author{
The $\mathrm{H1}$ and ZEUS Collaborations
}

F.D. Aaron ${ }^{14, e}$, H. Abramowicz ${ }^{72, a s}$, I. Abt ${ }^{57}$, L. Adamczyk ${ }^{36}$, M. Adamus ${ }^{85}$, R. Aggarwal ${ }^{15, m}$, C. Alexa $^{14}$, V. Andreev ${ }^{54}$, S. Antonelli ${ }^{11}$, P. Antonioli ${ }^{10}$, A. Antonov ${ }^{55}$, M. Arneodo ${ }^{78}$, O. Arslan ${ }^{12}$, V. Aushev ${ }^{39,40, a k}$, Y. Aushev ${ }^{40, a k, a l}$, O. Bachynska ${ }^{30}$, S. Backovic ${ }^{65}$, A. Baghdasaryan ${ }^{87}$, S. Baghdasaryan ${ }^{87}$, A. Bamberger ${ }^{26}$, A.N. Barakbaev ${ }^{2}$, G. Barbagli ${ }^{24}$, G. Bari ${ }^{10}$, F. Barreiro ${ }^{50}$, E. Barrelet ${ }^{64}$, W. Bartel ${ }^{30}$, N. Bartosik ${ }^{30}$, D. Bartsch ${ }^{12}$,

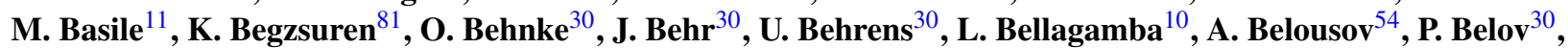
A. Bertolin ${ }^{61}$, S. Bhadra ${ }^{89}$, M. Bindi ${ }^{11}$, J.C. Bizot ${ }^{58}$, C. Blohm ${ }^{30}$, V. Bokhonov ${ }^{39, a k}$, K. Bondarenko ${ }^{40}$, E.G. Boos ${ }^{2}$, K. Borras ${ }^{30}$, D. Boscherini ${ }^{10}$, D. Bot ${ }^{30}$, V. Boudry ${ }^{63}$, I. Bozovic-Jelisavcic ${ }^{7}$, T. Bołd ${ }^{36}$, N. Brümmer ${ }^{17}$, J. Bracinik ${ }^{9}$, G. Brandt ${ }^{30}$, M. Brinkmann ${ }^{30}$, V. Brisson ${ }^{58}$, D. Britzger ${ }^{30}$, I. Brock ${ }^{12}$, E. Brownson ${ }^{49}$, R. Brugnera ${ }^{62}$, D. Bruncko ${ }^{35}$, A. Bruni ${ }^{10}$, G. Bruni ${ }^{10}$, B. Brzozowska ${ }^{84}$, A. Bunyatyan ${ }^{33,87}$, P.J. Bussey ${ }^{28}$,

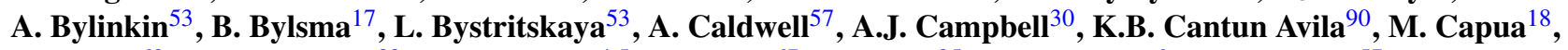
R. Carlin $^{62}$, C.D. Catterall ${ }^{89}$, F. Ceccopieri ${ }^{4,5}$, K. Cerny ${ }^{67}$, V. Cerny ${ }^{35}$, S. Chekanov ${ }^{6}$, V. Chekelian ${ }^{57}$, J. Chwastowski $^{19,0}$, J. Ciborowski ${ }^{84, a w}$, R. Ciesielski ${ }^{30, r}$, L. Cifarelli ${ }^{11}$, F. Cindolo ${ }^{10}$, A. Contin $^{11}$, J.G. Contreras ${ }^{90}$, A.M. Cooper-Sarkar ${ }^{59}$, N. Coppola ${ }^{30, \mathrm{~s}}$, M. Corradi ${ }^{10}$, F. Corriveau ${ }^{52}$, M. Costa ${ }^{77}$,

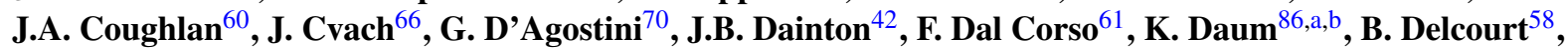
J. Delvax ${ }^{4,5}$, R.K. Dementiev ${ }^{56}$, M. Derrick ${ }^{6}$, R.C.E. Devenish ${ }^{59}$, S. De Pasquale ${ }^{11, k}$, E.A. De Wolf ${ }^{4,5}$, J. del Peso ${ }^{50}$, C. Diaconu ${ }^{51}$, M. Dobre ${ }^{29, g, h}$, D. Dobur ${ }^{26, a d}$, V. Dodonov ${ }^{33}$, B.A. Dolgoshein ${ }^{55, \dagger}$, G. Dolinska ${ }^{40}$, A. Dossanov $^{29,57}$, A.T. Doyle ${ }^{28}$, V. Drugakov ${ }^{91}$, A. Dubak ${ }^{65}$, L.S. Durkin ${ }^{17}$, S. Dusini ${ }^{61}$, G. Eckerlin ${ }^{30}$, S. Egli ${ }^{83}$, Y. Eisenberg ${ }^{68}$,

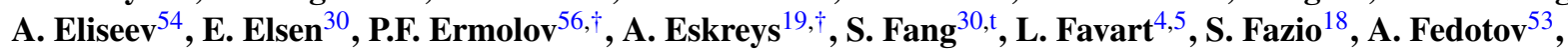
R. Felst ${ }^{30}$, J. Feltesse ${ }^{27}$, J. Ferencei ${ }^{35}$, J. Ferrando ${ }^{28}$, M.I. Ferrero ${ }^{77}$, J. Figiel ${ }^{19}$, D.-J. Fischer ${ }^{30}$, M. Fleischer ${ }^{30}$, A. Fomenko ${ }^{54}$, M. Forrest $^{28, a g}$, B. Foster ${ }^{59, \text { ao }}$, E. Gabathuler ${ }^{42}$, G. Gach ${ }^{36}$, A. Galas ${ }^{19}$, E. Gallo ${ }^{24}$, A. Garfagnini ${ }^{62}$, J. Gayler ${ }^{30}$, A. Geiser ${ }^{30}$, S. Ghazaryan ${ }^{30}$, I. Gialas ${ }^{16, \text { ah }}$, A. Gizhko ${ }^{40, \text { am }}$, L.K. Gladilin ${ }^{56, \text { an }}$, D. Gladkov ${ }^{55}$, C. Glasman ${ }^{50}$, A. Glazov ${ }^{30}$, L. Goerlich ${ }^{19}$, N. Gogitidze ${ }^{54}$, O. Gogota ${ }^{40}$, Y.A. Golubkov ${ }^{56}$, P. Göttlicher $^{30, u}$, M. Gouzevitch ${ }^{30, c}$, C. Grab ${ }^{92}$, I. Grabowska-Bołd ${ }^{36}$, A. Grebenyuk ${ }^{30}$, J. Grebenyuk ${ }^{30}$, T. Greenshaw ${ }^{42}$,

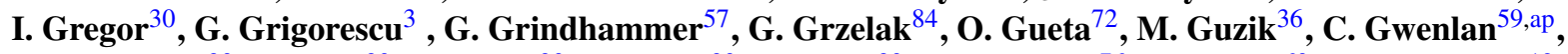

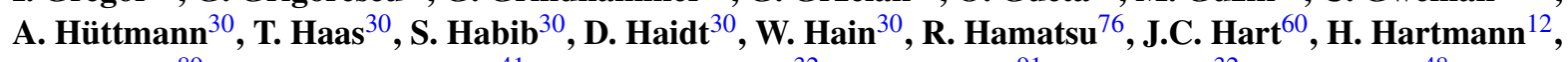
G. Hartner $^{89}$, R.C.W. Henderson ${ }^{41}$, E. Hennekemper ${ }^{32}$, H. Henschel ${ }^{91}$, M. Herbst ${ }^{32}$, G. Herrera ${ }^{48}$, M. Hildebrandt ${ }^{83}$, E. Hilger ${ }^{12}$, K.H. Hiller ${ }^{91}$, J. Hladkýy ${ }^{66}$, D. Hochman ${ }^{68}$, D. Hoffmann ${ }^{51}$, R. Hori ${ }^{75}$, R. Horisberger ${ }^{83}$, T. Hreus ${ }^{4,5}$, F. Huber ${ }^{31}$, Z.A. Ibrahim ${ }^{37}$, Y. Iga ${ }^{73}$, R. Ingbir ${ }^{72}$, M. Ishitsuka ${ }^{74}$, M. Jacquet $^{58}$, H.-P. Jakob ${ }^{12}$, X. Janssen ${ }^{4,5}$, F. Januschek ${ }^{30}$, T.W. Jones ${ }^{45}$, L. Jönsson ${ }^{47}$, M. Jüngst ${ }^{12}$, H. Jung ${ }^{30,4,5}$, I. Kadenko ${ }^{40}$, B. Kahle ${ }^{30}$, S. Kananov ${ }^{72}$, T. Kanno ${ }^{74}$, M. Kapichine ${ }^{23}$, U. Karshon ${ }^{68}$, F. Karstens ${ }^{26, a e}$, I.I. Katkov ${ }^{30, v}$, P. Kaur ${ }^{15, m}$, M. Kaur ${ }^{15}$, I.R. Kenyon ${ }^{9}$, A. Keramidas ${ }^{3}$, L.A. Khein ${ }^{56}$, C. Kiesling ${ }^{57}$, J.Y. Kim ${ }^{38}$, D. Kisielewska ${ }^{36}$, S. Kitamura ${ }^{76, a u}$, R. Klanner ${ }^{29}$, M. Klein ${ }^{42}$, U. Klein ${ }^{30, w}$, C. Kleinwort ${ }^{30}$, E. Koffeman ${ }^{3}$, R. Kogler ${ }^{29}$, N. Kondrashova ${ }^{40, \mathrm{am}}$, O. Kononenko ${ }^{40}$, P. Kooijman ${ }^{3}$, I. Korol $^{40}$, I.A. Korzhavina ${ }^{56, \text { an }}$, P. Kostka ${ }^{91}$, A. Kotański ${ }^{20, p}$, U. Kötz ${ }^{30}$, H. Kowalski ${ }^{30}$, M. Krämer $^{30}$, J. Kretzschmar ${ }^{42}$, K. Krüger ${ }^{32}$, O. Kuprash $^{30}$, M. Kuze ${ }^{74}$, M.P.J. Landon ${ }^{43}$, W. Lange ${ }^{91}$, G. Laštovička-Medin ${ }^{65}$, P. Laycock ${ }^{42}$, A. Lebedev ${ }^{54}$, A. Lee ${ }^{17}$, V. Lendermann ${ }^{32}$, B.B. Levchenko ${ }^{56}$, S. Levonian ${ }^{30}$, A. Levy ${ }^{72}$, V. Libov ${ }^{30}$, S. Limentani ${ }^{62}$, T.Y. Ling ${ }^{17}$, K. Lipka ${ }^{30, g}$, M. Lisovyi ${ }^{30}$, B. List ${ }^{30}$, J. List ${ }^{30}$, E. Lobodzinska ${ }^{30}$, B. Lobodzinski ${ }^{30}$, W. Lohmann ${ }^{91}$, B. Löhr ${ }^{30}$, E. Lohrmann ${ }^{29}$, K.R. Long ${ }^{44}$, A. Longhin ${ }^{61, a q}$, D. Lontkovskyi ${ }^{30}$, R. Lopez-Fernandez ${ }^{48}$, V. Lubimov ${ }^{53}$, O.Y. Lukina ${ }^{56}$,

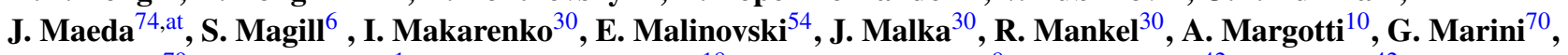
J.F. Martin ${ }^{79}$, H.-U. Martyn ${ }^{1}$, A. Mastroberardino ${ }^{18}$, M.C.K. Mattingly ${ }^{8}$, S.J. Maxfield ${ }^{42}$, A. Mehta ${ }^{42}$, I.-A. Melzer-Pellmann ${ }^{30}$, S. Mergelmeyer ${ }^{12}$, A.B. Meyer $^{30}$, H. Meyer ${ }^{86}$, J. Meyer $^{30}$, S. Miglioranzi ${ }^{30, x}$, 
S. Mikocki' ${ }^{19}$, I. Milcewicz-Mika ${ }^{19}$, F. Mohamad Idris ${ }^{37}$, V. Monaco ${ }^{77}$, A. Montanari ${ }^{30}$, F. Moreau ${ }^{63}$, A. Morozov $^{23}$, J.V. Morris ${ }^{60}$, J.D. Morris ${ }^{13,1}$, K. Mujkic ${ }^{30, y}$, K. Müller ${ }^{93}$, B. Musgrave ${ }^{6}$, K. Nagano ${ }^{80}$,

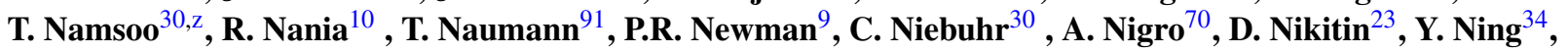

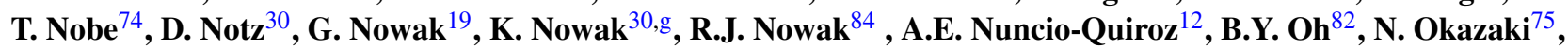
K. Olkiewicz ${ }^{19}$, J.E. Olsson ${ }^{30}$, Y. Onishchuk ${ }^{40}$, D. Ozerov ${ }^{30}$, P. Pahl ${ }^{30}$, V. Palichik ${ }^{23}$, M. Pandurovic ${ }^{7}$, K. Papageorgiu ${ }^{16}$, A. Parenti ${ }^{30}$, C. Pascaud ${ }^{58}$, G.D. Patel ${ }^{42}$, E. Paul ${ }^{12}$, J.M. Pawlak ${ }^{84}$, B. Pawlik ${ }^{19}$, P.G. Pelfer ${ }^{25}$,

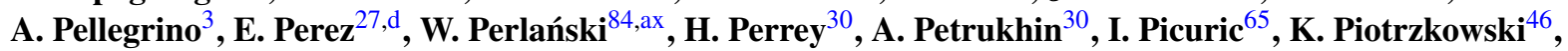
H. Pirumov ${ }^{31}$, D. Pitzl ${ }^{30}$, R. Plačakyte ${ }^{30, \mathrm{~g}}$, P. Pluciński ${ }^{85, \text { ay }}$, B. Pokorny ${ }^{67}$, N.S. Pokrovskiy ${ }^{2}$, R. Polifka ${ }^{67, \text {, }}$,

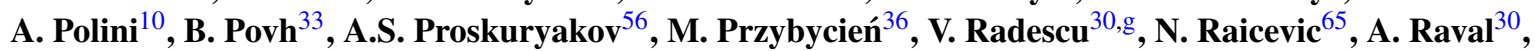
T. Ravdandorj ${ }^{81}$, D.D. Reeder ${ }^{49}$, P. Reimer ${ }^{66}$, B. Reisert $^{57}$, Z. $\operatorname{Ren}^{34}$, J. $\operatorname{Repond}^{6}$, Y.D. Ri ${ }^{76, a v}$, E. Rizvi $^{43}$, A. Robertson ${ }^{59}$, P. Robmann ${ }^{93}$, P. Roloff ${ }^{30, x}$, R. Roosen ${ }^{4,5}$, A. Rostovtsev ${ }^{53}$, M. Rotaru ${ }^{14}$, I. Rubinsky ${ }^{30}$, J.E. Ruiz Tabasco ${ }^{90}$, S. Rusakov ${ }^{54}$, M. Ruspa ${ }^{78}$, R. Sacchi $^{77}$, D. Š́lek ${ }^{67}$, U. Samson ${ }^{12}$, D.P.C. Sankey ${ }^{60}$, G. Sartorelli ${ }^{11}$, M. Sauter ${ }^{31}$, E. Sauvan ${ }^{51, j}$, A.A. Savin $^{49}$, D.H. Saxon ${ }^{28}$, M. Schioppa ${ }^{18}$, S. Schlenstedt ${ }^{91}$, P. Schleper ${ }^{29}$, W.B. Schmidke ${ }^{57}$, S. Schmitt ${ }^{30}$, U. Schneekloth ${ }^{30}$, L. Schoeffel ${ }^{27}$, V. Schönberg ${ }^{12}$, A. Schöning ${ }^{31}$, T. Schörner-Sadenius ${ }^{30}$, H.-C. Schultz-Coulon ${ }^{32}$, J. Schwartz ${ }^{52}$, F. Sciulli ${ }^{34}$, F. Sefkow ${ }^{30}$, L.M. Shcheglova ${ }^{56}$, R. Shehzadi ${ }^{12}$, S. Shimizu ${ }^{75, x}$, L.N. Shtarkov ${ }^{54}$, S. Shushkevich ${ }^{30}$, I. Singh ${ }^{15, \mathrm{~m}}$, I.O. Skillicorn ${ }^{28}$, W. Słomiński ${ }^{20, q}$, T. Sloan ${ }^{41}$, W.H. Smith ${ }^{49}$, V. Sola ${ }^{29}$, A. Solano ${ }^{77}$, Y. Soloviev ${ }^{26,54}$, D. Son ${ }^{21}$, P. Sopicki ${ }^{19}$, V. Sosnovtsev ${ }^{55}$, D. South ${ }^{30}$, V. Spaskov ${ }^{23}$, A. Specka ${ }^{63}$, A. Spiridonov ${ }^{30, a a}$, H. Stadie ${ }^{29}$, L. Stanco ${ }^{61}$, Z. Staykova ${ }^{4,5}$, M. Steder ${ }^{30}$, N. Stefaniuk ${ }^{40}$, B. Stella ${ }^{69}$, A. Stern ${ }^{72}$, T.P. Stewart ${ }^{79}$, A. Stifutkin ${ }^{55}$, G. Stoicea ${ }^{14}$, P. Stopa ${ }^{19}$, U. Straumann ${ }^{93}$, S. Suchkov ${ }^{55}$, G. Susinno ${ }^{18}$, L. Suszycki ${ }^{36}$, T. Sykora ${ }^{4,5,67}$, J. Sztuk-Dambietz ${ }^{29}$, J. Szuba ${ }^{30, a b}$, D. Szuba ${ }^{29}$, A.D. Tapper ${ }^{44}$, E. Tassi ${ }^{18, \mathrm{n}}$, J. Terrón ${ }^{50}$, T. Theedt ${ }^{30}$, P.D. Thompson ${ }^{9}$, H. Tiecke ${ }^{3}$,

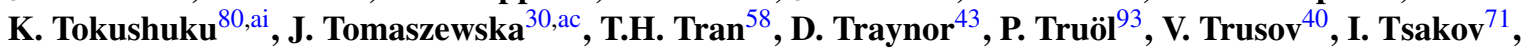

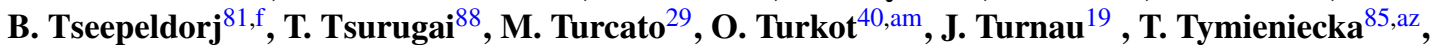
M. Vázquez ${ }^{3, x}$, A. Valkárová ${ }^{67}$, C. Vallée ${ }^{51}$, P. Van Mechelen $^{4,5}$, Y. Vazdik $^{54}$, A. Verbytskyi $^{30}$, O. Viazlo $^{40}$, N.N. Vlasov ${ }^{26, \text { af }}$, R. Walczak ${ }^{59}$, W.A.T. Wan Abdullah ${ }^{37}$, D. Wegener $^{22}$, J.J. Whitmore ${ }^{82, a r}$, K. Wichmann ${ }^{30}$,

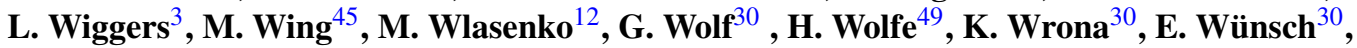

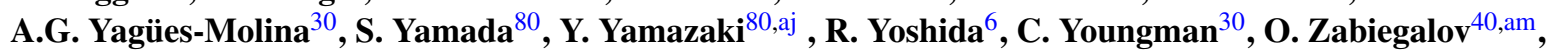
J. Žáček ${ }^{67}$, J. Zálešák ${ }^{66}$, L. Zawiejskii ${ }^{19}$, O. Zenaiev ${ }^{30}$, W. Zeuner ${ }^{30, x}$, Z. Zhang ${ }^{58}$, B.O. Zhautykov ${ }^{2}$, N. Zhmak ${ }^{39, a k}$, A. Zhokin ${ }^{53}$, A. Zichichii ${ }^{11}$, R. Žlebčík ${ }^{67}$, H. Zohrabyan ${ }^{87}$, Z. Zolkapli ${ }^{37}$, F. Zomer ${ }^{58}$, D.S. Zotkin ${ }^{56}$, A.F. $\dot{Z}^{2}{ }^{3}{ }^{84}$

\footnotetext{
${ }^{1}$ I. Physikalisches Institut der RWTH, Aachen, Germany

${ }^{2}$ Institute of Physics and Technology of Ministry of Education and Science of Kazakhstan, Almaty, Kazakhstan

${ }^{3}$ NIKHEF and University of Amsterdam, Amsterdam, Netherlands ${ }^{c a}$

${ }^{4}$ Inter-University Institute for High Energies ULB-VUB, Brussels, Belgium ${ }^{\text {bb }}$

${ }^{5}$ Universiteit Antwerpen, Antwerpen, Belgium ${ }^{\text {bb }}$

${ }^{6}$ Argonne National Laboratory, Argonne, IL 60439-4815, USA

${ }^{7}$ Vinca Institute of Nuclear Sciences, University of Belgrade, 1100 Belgrade, Serbia

${ }^{8}$ Andrews University, Berrien Springs, MI 49104-0380, USA

${ }^{9}$ School of Physics and Astronomy, University of Birmingham, Birmingham, UK ${ }^{\text {bo }}$

${ }^{10}$ INFN Bologna, Bologna, Italy ${ }^{\text {bm }}$

${ }^{11}$ University and INFN Bologna, Bologna, Italy ${ }^{\mathrm{bm}}$

${ }^{12}$ Physikalisches Institut der Universität Bonn, Bonn, Germany ${ }^{\text {bn }}$

${ }^{13}$ H.H. Wills Physics Laboratory, University of Bristol, Bristol, UK ${ }^{\text {bo }}$

${ }^{14}$ National Institute for Physics and Nuclear Engineering (NIPNE), Bucharest, Romania ${ }^{\text {bj }}$

${ }^{15}$ Department of Physics, Panjab University, Chandigarh, India

${ }^{16}$ Department of Engineering in Management and Finance, Univ. of the Aegean, Chios, Greece

${ }^{17}$ Physics Department, Ohio State University, Columbus, OH 43210, USA ${ }^{\text {bl }}$

${ }^{18}$ Physics Department and INFN, Calabria University, Cosenza, Italy ${ }^{\mathrm{bm}}$

${ }^{19}$ The Henryk Niewodniczanski Institute of Nuclear Physics, Polish Academy of Sciences, Cracow, Poland ${ }^{\text {bc }}$

${ }^{20}$ Department of Physics, Jagellonian University, Cracow, Poland

${ }^{21}$ Center for High Energy Physics, Kyungpook National University, Daegu, South Korea ${ }^{\text {bu }}$

${ }^{22}$ Institut für Physik, TU Dortmund, Dortmund, Germany ${ }^{\text {ba }}$

${ }^{23}$ Joint Institute for Nuclear Research, Dubna, Russia

${ }^{24}$ INFN Florence, Florence, Italy ${ }^{\mathrm{bm}}$

${ }^{25}$ University and INFN Florence, Florence, Italy ${ }^{\text {bm }}$
} 
${ }^{26}$ Fakultät für Physik der Universität Freiburg i.Br., Freiburg i.Br., Germany

${ }^{27} \mathrm{CEA}, \mathrm{DSM} / \mathrm{Irfu}, \mathrm{CE}-$ Saclay, Gif-sur-Yvette, France

${ }^{28}$ School of Physics and Astronomy, University of Glasgow, Glasgow, UK ${ }^{\text {bo }}$

${ }^{29}$ Institut für Experimentalphysik, Universität Hamburg, Hamburg, Germany ba,bs

${ }^{30}$ Deutsches Elektronen-Synchrotron DESY, Hamburg, Germany

${ }^{31}$ Physikalisches Institut, Universität Heidelberg, Heidelberg, Germany ${ }^{\text {ba }}$

${ }^{32}$ Kirchhoff-Institut für Physik, Universität Heidelberg, Heidelberg, Germany ${ }^{\text {ba }}$

${ }^{33}$ Max-Planck-Institut für Kernphysik, Heidelberg, Germany

${ }^{34}$ Nevis Laboratories, Columbia University, Irvington on Hudson, NY 10027, USA ${ }^{\text {bq }}$

${ }^{35}$ Institute of Experimental Physics, Slovak Academy of Sciences, Košice, Slovak Republic ${ }^{\text {bd }}$

${ }^{36}$ Faculty of Physics and Applied Computer Science, AGH-University of Science and Technology, Krakow, Poland ${ }^{\text {br }}$

${ }^{37}$ Jabatan Fizik, Universiti Malaya, 50603 Kuala Lumpur, Malaysiabp

${ }^{38}$ Institute for Universe and Elementary Particles, Chonnam National University, Kwangju, South Korea

${ }^{39}$ Institute for Nuclear Research, National Academy of Sciences, Kyiv, Ukraine

${ }^{40}$ Department of Nuclear Physics, National Taras Shevchenko University of Kyiv, Kyiv, Ukraine

${ }^{41}$ Department of Physics, University of Lancaster, Lancaster, UK ${ }^{\text {bo }}$

${ }^{42}$ Department of Physics, University of Liverpool, Liverpool, UK ${ }^{\text {bo }}$

${ }^{43}$ School of Physics and Astronomy, Queen Mary, University of London, London, UK ${ }^{\text {bo }}$

${ }^{44}$ High Energy Nuclear Physics Group, Imperial College London, London, UK ${ }^{\text {bo }}$

${ }^{45}$ Physics and Astronomy Department, University College London, London, $\mathrm{UK}^{\text {bo }}$

${ }^{46}$ Institut de Physique Nucléaire, Université Catholique de Louvain, Louvain-la-Neuve, Belgium ${ }^{\text {bv }}$

${ }^{47}$ Physics Department, University of Lund, Lund, Sweden ${ }^{\text {be }}$

${ }^{48}$ Departamento de Fisica, CINVESTAV IPN, México City, México ${ }^{\text {bh }}$

${ }^{49}$ Department of Physics, University of Wisconsin, Madison, WI 53706, USA ${ }^{\text {bl }}$

${ }_{50}^{50}$ Departamento de Física Teórica, Universidad Autónoma de Madrid, Madrid, Spain ${ }^{\text {bw }}$

${ }^{51}$ CPPM, Aix-Marseille Univ, CNRS/IN2P3, 13288 Marseille, France

${ }^{52}$ Department of Physics, McGill University, Montréal, Québec H3A 2T8, Canada ${ }^{\text {bx }}$

${ }^{53}$ Institute for Theoretical and Experimental Physics, Moscow, Russia ${ }^{\text {bi }}$

${ }^{54}$ Lebedev Physical Institute, Moscow, Russia

${ }_{55}^{55}$ Moscow Engineering Physics Institute, Moscow, Russia ${ }^{\text {by }}$

${ }^{56}$ Skobeltsyn Institute of Nuclear Physics, Lomonosov Moscow State University, Moscow, Russia ${ }^{\text {bz }}$

${ }^{57}$ Max-Planck-Institut für Physik, Munich, Germany

${ }^{58}$ LAL, Université Paris-Sud, CNRS/IN2P3, Orsay, France

${ }^{59}$ Department of Physics, University of Oxford, Oxford, UK ${ }^{\text {bo }}$

${ }^{60}$ STFC, Rutherford Appleton Laboratory, Didcot, Oxfordshire, $\mathrm{UK}^{\text {bo }}$

${ }^{61}$ INFN Padova, Padova, Italy ${ }^{\mathrm{bm}}$

${ }^{62}$ 'Dipartimento di Fisica dell' Università and INFN, Padova, Italy ${ }^{b m}$

${ }^{63}$ LLR, Ecole Polytechnique, CNRS/IN2P3, Palaiseau, France

${ }^{64}$ LPNHE, Université Pierre et Marie Curie Paris 6, Université Denis Diderot Paris 7, CNRS/IN2P3, Paris, France

${ }^{65}$ Faculty of Science, University of Montenegro, Podgorica, Montenegro ${ }^{\text {bk }}$

${ }^{66}$ Institute of Physics of the Academy of Sciences of the Czech Republic, Praha, Czech Republic ${ }^{b f}$

${ }^{67}$ Faculty of Mathematics and Physics of Charles University, Praha, Czech Republic ${ }^{\text {bf }}$

${ }^{68}$ Department of Particle Physics and Astrophysics, Weizmann Institute, Rehovot, Israel

${ }^{69}$ Dipartimento di Fisica, Università di Roma Tre and INFN Roma 3, Rome, Italy

${ }^{70}$ Dipartimento di Fisica, Università'La Sapienza' and INFN, Rome, Italy bm

${ }^{71}$ Institute for Nuclear Research and Nuclear Energy, Sofia, Bulgaria

${ }_{72}^{72}$ Raymond and Beverly Sackler Faculty of Exact Sciences, School of Physics, Tel Aviv University, Tel Aviv, Israel ${ }^{\text {cb }}$

${ }^{73}$ Polytechnic University, Tokyo, Japan ${ }^{\text {bt }}$

${ }_{75}^{74}$ Department of Physics, Tokyo Institute of Technology, Tokyo, Japan ${ }^{\text {bt }}$

${ }_{76}^{75}$ Department of Physics, University of Tokyo, Tokyo, Japan ${ }^{\text {bt }}$

${ }^{76}$ Department of Physics, Tokyo Metropolitan University, Tokyo, Japan ${ }^{\text {bt }}$

${ }^{77}$ Università di Torino and INFN, Torino, Italy ${ }^{\mathrm{bm}}$

${ }^{78}$ Università del Piemonte Orientale, Novara, and INFN, Torino, Italy ${ }^{\text {bm }}$

${ }^{79}$ Department of Physics, University of Toronto, Toronto, Ontario M5S 1A7, Canada ${ }^{\text {bx }}$

${ }^{80}$ Institute of Particle and Nuclear Studies, KEK, Tsukuba, Japan ${ }^{\text {bt }}$

${ }^{81}$ Institute of Physics and Technology of the Mongolian Academy of Sciences, Ulaanbaatar, Mongolia

${ }^{82}$ Department of Physics, Pennsylvania State University, University Park, PA 16802, USA ${ }^{\text {bq }}$

${ }^{83}$ Paul Scherrer Institut, Villigen, Switzerland

${ }^{84}$ Faculty of Physics, University of Warsaw, Warsaw, Poland

${ }^{85}$ National Centre for Nuclear Research, Warsaw, Poland

${ }^{86}$ Fachbereich C, Universität Wuppertal, Wuppertal, Germany

${ }^{87}$ Yerevan Physics Institute, Yerevan, Armenia

${ }^{88}$ Faculty of General Education, Meiji Gakuin University, Yokohama, Japan ${ }^{\text {bt }}$

${ }^{89}$ Department of Physics, York University, Toronto, Ontario M3J 1P3, Canada ${ }^{\text {bx }}$

${ }^{90}$ Departamento de Fisica Aplicada, CINVESTAV, Mérida, Yucatán, México ${ }^{\text {bh }}$ 
${ }^{91}$ Deutsches Elektronen-Synchrotron DESY, Zeuthen, Germany

${ }^{92}$ Institut für Teilchenphysik, ETH, Zurich, Switzerland ${ }^{\text {bg }}$

${ }^{93}$ Physik-Institut der Universität Zürich, Zurich, Switzerland ${ }^{\text {bg }}$

Received: 20 July 2012 / Revised: 24 September 2012 / Published online: 10 October 2012

(C) The Author(s) 2012. This article is published with open access at Springerlink.com

\begin{abstract}
A combination of the inclusive diffractive cross section measurements made by the $\mathrm{H} 1$ and ZEUS Collaborations at HERA is presented. The analysis uses samples of diffractive deep inelastic ep scattering data at a centreof-mass energy $\sqrt{s}=318 \mathrm{GeV}$ where leading protons are detected by dedicated spectrometers. Correlations of systematic uncertainties are taken into account, resulting in an
\end{abstract}

a e-mail: daum@mail.desy.de

${ }^{b}$ Also at Rechenzentrum, Universität Wuppertal, Wuppertal, Germany.

${ }^{\mathrm{c}}$ Also at IPNL, Université Claude Bernard Lyon 1, CNRS/IN2P3, Villeurbanne, France.

${ }^{\mathrm{d}}$ Also at CERN, Geneva, Switzerland.

${ }^{\mathrm{e}}$ Also at Faculty of Physics, University of Bucharest, Bucharest, Romania.

${ }^{\mathrm{f}}$ Also at Ulaanbaatar University, Ulaanbaatar, Mongolia.

${ }^{\mathrm{g}}$ Supported by the Initiative and Networking Fund of the Helmholtz Association (HGF) under the contract VH-NG-401 and S0-072.

${ }^{\mathrm{h}}$ Absent on leave from NIPNE-HH, Bucharest, Romania.

${ }^{\mathrm{i}}$ Also at Department of Physics, University of Toronto, Toronto, Ontario, Canada M5S 1A7.

jAlso at LAPP, Université de Savoie, CNRS/IN2P3, Annecy-le-Vieux, France.

${ }^{k}$ Now at University of Salerno, Italy.

${ }^{l}$ Now at Queen Mary University of London, UK.

${ }^{m}$ Also funded by Max Planck Institute for Physics, Munich, Germany. ${ }^{n}$ Also Senior Alexander von Humboldt Research Fellow at Hamburg University, Institute of Experimental Physics, Hamburg, Germany.

${ }^{\circ}$ Also at Cracow University of Technology, Faculty of Physics, Mathemathics and Applied Computer Science, Poland.

${ }^{\mathrm{p}}$ Supported by the research grant No. 1 P03B 04529 (2005-2008).

${ }^{\mathrm{q}}$ Supported by the Polish National Science Centre, project No. DEC2011/01/BST2/03643.

${ }^{r}$ Now at Rockefeller University, New York, NY 10065, USA.

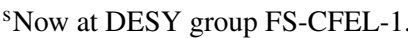

${ }^{t}$ Now at Institute of High Energy Physics, Beijing, China.

${ }^{u}$ Now at DESY group FEB, Hamburg, Germany.

${ }^{v}$ Also at Moscow State University, Russia.

${ }^{\mathrm{w}}$ Now at University of Liverpool, UK.

${ }^{\mathrm{x}}$ Now at CERN, Geneva, Switzerland.

${ }^{y}$ Also affiliated with Universtiy College London, UK.

${ }^{\mathrm{z}}$ Now at Goldman Sachs, London, UK.

${ }^{\text {aa }}$ Also at Institute of Theoretical and Experimental Physics, Moscow, Russia.

${ }^{\mathrm{ab}}$ Also at FPACS, AGH-UST, Cracow, Poland.

${ }^{a c}$ Partially supported by Warsaw University, Poland. improved precision of the cross section measurement which reaches $6 \%$ for the most precise points. The combined data cover the range $2.5<Q^{2}<200 \mathrm{GeV}^{2}$ in photon virtuality, $0.00035<x_{\mathbb{P}}<0.09$ in proton fractional momentum loss, $0.09<|t|<0.55 \mathrm{GeV}^{2}$ in squared four-momentum transfer at the proton vertex and $0.0018<\beta<0.816$ in $\beta=x / x_{\mathbb{P}}$, where $x$ is the Bjorken scaling variable.

\footnotetext{
${ }^{\text {ad }}$ Now at Istituto Nucleare di Fisica Nazionale (INFN), Pisa, Italy.

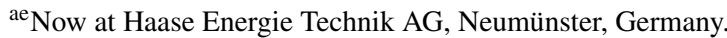

${ }^{a f}$ Now at Department of Physics, University of Bonn, Germany.

${ }^{\text {ag }}$ Now at Biodiversität und Klimaforschungszentrum (BiK-F), Frankfurt, Germany.

ah Also affiliated with DESY, Germany.

ai Also at University of Tokyo, Japan.

${ }^{a j}$ Now at Kobe University, Japan.

${ }^{\mathrm{ak}}$ Supported by DESY, Germany.

${ }^{a l}$ Member of National Technical University of Ukraine, Kyiv Polytech-
} nic Institute, Kyiv, Ukraine.

${ }^{a m}$ Member of National University of Kyiv-Mohyla Academy, Kyiv, Ukraine.

an Partly supported by the Russian Foundation for Basic Research, grant 11-02-91345-DFG_a.

${ }^{\text {ao }}$ Alexander von Humboldt Professor; also at DESY and University of Oxford.

ap STFC Advanced Fellow.

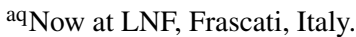

${ }^{\text {ar }}$ This material was based on work supported by the National Science Foundation, while working at the Foundation.

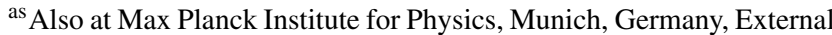
Scientific Member.

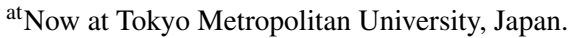

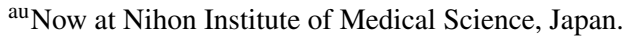

${ }^{\text {av }}$ Now at Osaka University, Osaka, Japan.

${ }^{\text {aw }}$ Also at Lodz University, Poland.

${ }^{\text {ax }}$ Member of Lodz University, Poland.

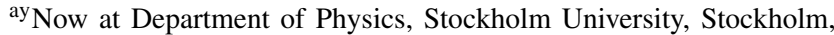
Sweden

${ }^{a z}$ Also at Cardinal Stefan Wyszyński University, Warsaw, Poland.

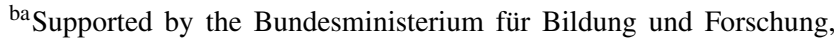
FRG, under contract numbers 05H09GUF, 05H09VHC, 05H09VHF, 05H16PEA.

${ }^{\mathrm{bb}}$ Supported by FNRS-FWO-Vlaanderen, IISN-IIKW and IWT and by Interuniversity Attraction Poles Programme, Belgian Science Policy.

${ }^{b c}$ Supported by Polish Ministry of Science and Higher Education, grants DPN/N168/DESY/2009 and DPN/N188/DESY/2009. 


\section{Introduction}

Diffractive collisions in deep inelastic electron-proton scattering (DIS), $e p \rightarrow e X p$, where the proton in the final state carries most of the beam momentum and $X$ represents all other final state particles, have been studied extensively at the HERA collider. They can be viewed as resulting from processes in which a photon exchanged between the electron and the proton probes a colour-singlet combination of partons with vacuum quantum numbers emitted by the proton. The negative four-momentum squared of the virtual photon, $Q^{2}$, supplies a hard scale, which allows the application of perturbative quantum chromodynamics (QCD). Diffractive reactions in DIS are a tool to investigate low-momentum partons in the proton, notably through the study of diffractive parton distribution functions (DPDFs), determined by a QCD analysis of the data.

In diffractive $e p$ scattering the virtual photon dissociates at a photon-proton centre-of-mass energy $W$ and squared four-momentum transfer $t$ at the proton vertex (Fig. 1), producing a hadronic system $X$ with mass $M_{X}$. The fractional longitudinal momentum loss of the proton is denoted as $x_{\mathbb{P}}$, while the fraction of this momentum taking part in the interaction with the photon is denoted as $\beta$. These variables are related to Bjorken $x$ by $x=\beta x_{\mathbb{P}}$. The variable $\beta$ is related to $M_{X}, t$ and $Q^{2}$ by $\beta=Q^{2} /\left(Q^{2}+M_{X}^{2}-t\right)$. The variable $x_{\mathbb{P}}$ is given by $x_{\mathbb{P}}=\left(Q^{2}+M_{X}^{2}-t\right) /\left(Q^{2}+W^{2}-m_{p}^{2}\right)$, where

\footnotetext{
${ }^{\text {bd }}$ Supported by VEGA SR grant no. 2/7062/27.

${ }^{\text {be }}$ Supported by the Swedish Natural Science Research Council.

${ }^{\text {bf }}$ Supported by the Ministry of Education of the Czech Republic under the projects LC527, INGO-LA09042 and MSM0021620859.

${ }^{b g}$ Supported by the Swiss National Science Foundation.

${ }^{\text {bh }}$ Supported by CONACYT, México, grant 48778-F.

${ }^{b i}$ Russian Foundation for Basic Research (RFBR), grant no. 1329.2008.2 and Rosatom.

${ }^{\text {bj }}$ Supported by the Romanian National Authority for Scientific Research under the contract PN 09370101.

${ }^{b k}$ Partially Supported by Ministry of Science of Montenegro, no. 051/3-3352.

${ }^{b l}$ Supported by the US Department of Energy.

${ }^{\text {bm }}$ Supported by the Italian National Institute for Nuclear Physics (INFN).

${ }^{\text {bn }}$ Supported by the German Federal Ministry for Education and Research (BMBF), under contract No. 05 H09PDF.

${ }^{\text {bo }}$ Supported by the Science and Technology Facilities Council, UK.

${ }^{\text {bp }}$ Supported by an FRGS grant from the Malaysian government.

${ }^{\mathrm{bq}}$ Supported by the US National Science Foundation. Any opinion, findings and conclusions or recommendations expressed in this material are those of the authors and do not necessarily reflect the views of the National Science Foundation.

${ }^{b r}$ Supported by the Polish Ministry of Science and Higher Education and its grants for Scientific Research.
}

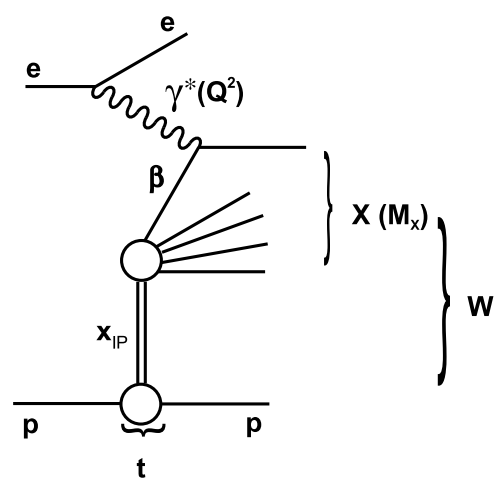

Fig. 1 Diagram of the reaction $e p \rightarrow e X p$

$m_{p}$ is the proton mass. The variables $W, Q^{2}$ and the fractional energy loss $y$ of the electron in the proton rest frame are related by $W^{2} \simeq s y-Q^{2}$, where $s$ is the square of the ep centre-of-mass energy.

Similarly to inclusive DIS, diffractive cross section measurements are conventionally expressed in terms of the reduced diffractive cross section, $\sigma_{r}^{D(4)}$, which is related to the measured $e p$ cross section by

$$
\begin{aligned}
\frac{\mathrm{d} \sigma^{e p \rightarrow e X p}}{\mathrm{~d} \beta \mathrm{d} Q^{2} \mathrm{~d} x_{\mathbb{P}} \mathrm{d} t}= & \frac{4 \pi \alpha^{2}}{\beta Q^{4}}\left[1-y+\frac{y^{2}}{2}\right] \\
& \times \sigma_{r}^{D(4)}\left(\beta, Q^{2}, x_{\mathbb{P}}, t\right) .
\end{aligned}
$$

The reduced cross section $\sigma_{r}{ }^{D(3)}\left(\beta, Q^{2}, x_{\mathbb{P}}\right)$ is obtained by

\footnotetext{
${ }^{\text {bs }}$ Supported by the German Federal Ministry for Education and Research (BMBF), under contract No. 05h09GUF, and the SFB 676 of the Deutsche Forschungsgemeinschaft (DFG).

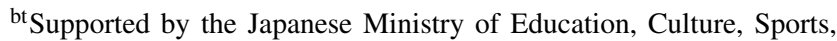
Science and Technology (MEXT) and its grants for Scientific Research.

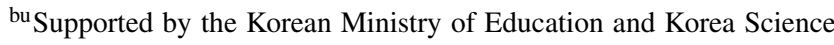
and Engineering Foundation.

${ }^{\text {bv }}$ Supported by FNRS and its associated funds (IISN and FRIA) and by an Inter-University Attraction Poles Programme subsidised by the Belgian Federal Science Policy Office.

${ }^{b w}$ Supported by the Spanish Ministry of Education and Science through funds provided by CICYT.

${ }^{\mathrm{bx}}$ Supported by the Natural Sciences and Engineering Research Council of Canada (NSERC).

by Partially supported by the German Federal Ministry for Education and Research (BMBF).

${ }^{\mathrm{bz}}$ Supported by RF Presidential grant N 4142.2010.2 for Leading Scientific Schools, by the Russian Ministry of Education and Science through its grant for Scientific Research on High Energy Physics and under contract No. 02.740.11.0244.

${ }^{c a}$ Supported by the Netherlands Foundation for Research on Matter (FOM).

${ }^{\mathrm{cb}}$ Supported by the Israel Science Foundation.

${ }^{\dagger}$ Deceased.
} 
integrating $\sigma_{r}{ }^{D(4)}\left(\beta, Q^{2}, x_{\mathbb{P}}, t\right)$ over $t$. The diffractive reduced cross section is related to the diffractive structure functions by:

$$
\begin{aligned}
& \sigma_{r}^{D(3)}\left(x_{\mathbb{P}}, \beta, Q^{2}, y\right) \\
& \quad=F_{2}^{D(3)}\left(x_{\mathbb{P}}, \beta, Q^{2}\right)-\frac{y^{2}}{1+(1-y)^{2}} F_{L}^{D(3)}\left(x_{\mathbb{P}}, \beta, Q^{2}\right) .
\end{aligned}
$$

Experimentally, diffractive $e p$ scattering is characterised by the presence of a leading proton in the final state and by a depletion of hadronic activity in the pseudo-rapidity ${ }^{1}$ distribution of particles (large rapidity gap, LRG) in the forward (proton) direction. Both of these signatures have been exploited in various analyses by $\mathrm{H} 1$ and ZEUS to select diffractive samples either by tagging the outgoing proton in dedicated proton spectrometers [1-4] or by requiring the presence of a large rapidity gap [4-6]. The two methods differ partially in the accessible kinematic ranges (lower $x_{\mathbb{P}}$ reach for the LRG data) and substantially in their dominant sources of systematic uncertainties. In LRG-based measurements, the largest uncertainty arises from proton dissociative events, $e p \rightarrow e X N$, in which the proton dissociates into a low mass state $N$. Low $x_{\mathbb{P}}$ samples selected by the proton spectrometers have little or no proton dissociation contribution, but their precision is limited statistically by the small acceptances and systematically by large uncertainties in the proton tagging efficiency, which strongly depends on the proton-beam optics. The results from both methods are found to be consistent $[1,2,4,6,7]$.

Combining measurements can provide more precise and kinematically extended data than the individual measurements. In this paper, a combination of the H1 $[1,2]$ and the ZEUS [3, 4] proton spectrometer results is presented. The combination is performed using the weighted averaging method introduced in [8] and extended in $[9,10]$. The correlated systematic uncertainties and global normalisations are constrained in the fit such that one consistent data set is obtained. Since H1 and ZEUS have employed different experimental techniques, using different detectors and methods of kinematic reconstruction, the combination leads to significantly reduced uncertainties. The kinematic range of the combined data is: $2.5 \leq Q^{2} \leq 200 \mathrm{GeV}^{2}, 0.0018 \leq \beta \leq$ $0.816,0.00035 \leq x_{\mathbb{P}} \leq 0.09$ and $0.09<|t|<0.55 \mathrm{GeV}^{2}$. The latter range restricts the analysis to the $t$ values directly accessible by both the H1 and ZEUS proton spectrometers.

\footnotetext{
${ }^{1}$ The pseudo-rapidity is defined as $\eta=-\ln \tan \theta / 2$ where the polar angle $\theta$ is measured with respect to the proton beam direction.
}

\section{Combination of the H1 and ZEUS measurements}

\subsection{Data samples}

The H1 [11-13] and ZEUS [14] detectors were general purpose instruments which consisted of tracking systems surrounded by electromagnetic and hadronic calorimeters and muon detectors, ensuring close to $4 \pi$ coverage about the $e p$ interaction point. Both detectors were equipped with proton spectrometers; the Leading Proton Spectrometer (LPS) for ZEUS, the Forward Proton Spectrometer (FPS) and the Very Forward Proton Spectrometer (VFPS) for H1. The LPS and FPS spectrometers were located between 60 and $90 \mathrm{~m}$ away from the main detectors in the forward (proton beam) direction. The VFPS spectrometer was located around $220 \mathrm{~m}$ away from the main $\mathrm{H} 1$ detector in the forward direction.

The combination is based on the cross sections measured with the H1 FPS [1, 2] and the ZEUS LPS [3, 4]. The bulk of the data $[1,2,4]$ was taken at electron and proton beam energies of $E_{e} \simeq 27.5 \mathrm{GeV}$ and $E_{p}=920 \mathrm{GeV}$, respectively, corresponding to an $e p$ centre-of-mass energy of $\sqrt{s}=318 \mathrm{GeV}$. The earlier ZEUS LPS data [3] collected at $E_{p}=820 \mathrm{GeV}$ are corrected to a common $\sqrt{s}=$ $318 \mathrm{GeV}$ by using the extrapolation procedure described in Sect. 2.1.2. The three-fold differential reduced cross sections, $\sigma_{r}{ }^{D(3)}\left(\beta, Q^{2}, x_{\mathbb{P}}\right)$, are combined. For the original measurements, the main H1 and ZEUS detectors are used to reconstruct $Q^{2}, W$ and $x$, whereas $M_{X}, \beta, x_{\mathbb{P}}$ and $t$ are derived from the proton spectrometer measurements or from combined information of the proton spectrometers and the main detectors. In Table 1 the data sets used for the combination are listed together with their kinematic ranges and integrated luminosities.

\subsubsection{Restricted t range}

In the individual analyses [1-4] the reduced cross sections are directly measured for ranges of the squared fourmomentum transfer $t$ visible to the proton spectrometers (see Table 1) and extrapolated to the range ${ }^{2}\left|t_{\min }\right|<|t|<$ $1 \mathrm{GeV}^{2}$ (denoted in the following as 'the full $t$ range'), assuming an exponential $t$ dependence of the diffractive cross section and using the exponential slope measured from the data. Due to the uncertainties of the slope parameters measured by H1 [1, 2] and ZEUS [3, 4], this extrapolation introduces an additional uncertainty in the normalisation of the cross section. To reduce this source of systematic uncertainty, the $\mathrm{H} 1$ and ZEUS cross sections are combined in the restricted $t$ range $0.09<|t|<0.55 \mathrm{GeV}^{2}$ covered by the proton spectrometer acceptances of both detectors for

\footnotetext{
${ }^{2}$ The smallest kinematically accessible value of $|t|$ is denoted as $\left|t_{\min }\right|$.
} 
Table $1 \mathrm{H} 1$ and ZEUS data sets used for the combination of the measurements

\begin{tabular}{llllllll}
\hline Data Set & $\begin{array}{c}Q^{2} \text { range } \\
{\left[\mathrm{GeV}^{2}\right]}\end{array}$ & $x_{\mathbb{P}}$ range & $y$ range & $\beta$ range & $\begin{array}{l}t \text { range } \\
{\left[\mathrm{GeV}^{2}\right]}\end{array}$ & $\begin{array}{c}\text { Luminosity } \\
{\left[\mathrm{pb}^{-1}\right]}\end{array}$ & Ref. \\
\hline H1 FPS HERA II & $4-700$ & $<0.1$ & $0.03-0.8$ & $0.001-1$ & $0.1-0.7$ & 156.6 & {$[2]$} \\
H1 FPS HERA I & $2-50$ & $<0.1$ & $0.02-0.6$ & $0.004-1$ & $0.08-0.5$ & 28.4 & {$[1]$} \\
& & & $\begin{array}{l}W \text { range } \\
{[\mathrm{GeV}]}\end{array}$ & $\begin{array}{l}M_{X} \text { range } \\
{[\mathrm{GeV}]}\end{array}$ & & & \\
ZEUS LPS 2 & $2.5-120$ & $0.0002-0.1$ & $40-240$ & $2-40$ & $0.09-0.55$ & 32.6 & {$[4]$} \\
ZEUS LPS 1 & $2-100$ & $<0.1$ & $25-240$ & $>1.5$ & $0.075-0.35$ & 3.6 & {$[3]$} \\
\hline
\end{tabular}

the bulk of the data. The correction factors from the visible $t$ range of the 'FPS HERA I' and 'LPS 1' data samples to the restricted $t$ range are evaluated by using the $t$ dependencies as a function of $x_{\mathbb{P}}$ measured for each sample. The correction factors for the most precise 'FPS HERA II' data are applied in bins of $\beta, Q^{2}$ and $x_{\mathbb{P}}$. For the 'LPS 2' sample the restricted range coincides with the visible range. Because of the uncertainty on the exponential slope parameter, such factors introduce uncertainties of $2.2 \%, 1.1 \%$ and $5 \%$ on the 'FPS HERA II', 'FPS HERA I' and 'LPS 1' data, respectively, which are included in the normalisation uncertainty on each sample. The total normalisation uncertainties of the data samples are listed in Table 2. In the restricted $t$ range, these uncertainties are in general smaller and the average normalisations are in better agreement than in the full $t$ range; the ratio of the 'FPS HERA II' to the 'LPS 2' data averaged over the measured data points, which is $0.85 \pm$ 0.01 (stat) \pm 0.03 (sys) ${ }_{-0.12}^{+0.09}$ (norm) in the full $t$ range [2], becomes $0.91 \pm 0.01$ (stat) \pm 0.03 (sys) \pm 0.08 (norm) in the restricted $t$ range. Within the uncertainties, the ratio does not show any significant $\beta, Q^{2}$ or $x_{\mathbb{P}}$ dependence.

\subsubsection{Extrapolation to a common $\left(Q^{2}, x_{\mathbb{P}}, \beta\right)$ grid}

The original binning schemes of the $\sigma_{r}^{D(3)}$ measurements are very different for $\mathrm{H} 1$ and ZEUS. In the $\mathrm{H} 1$ case the measurements are extracted at fixed $\beta$, whereas for ZEUS the cross section is measured at fixed $M_{X}$; also the $Q^{2}$ and $x_{\mathbb{P}}$ central values differ. Therefore, prior to the combination, the $\mathrm{H} 1$ and ZEUS data are transformed to a common grid of

Table 2 Normalisation uncertainties in the full range $|t|<1 \mathrm{GeV}^{2}$ and in the restricted $t$ range for the data used for the combination of the measurements

\begin{tabular}{llc}
\hline Data Set & $\left|t_{\min }\right|<|t|<1 \mathrm{GeV}^{2}$ & $0.09<|t|<0.55 \mathrm{GeV}^{2}$ \\
\hline FPS HERA II & $\pm 6 \%$ & $\pm 5 \%$ \\
FPS HERA I & $\pm 10 \%$ & $\pm 10 \%$ \\
LPS 2 & $+11 \%,-7 \%$ & $\pm 7 \%$ \\
LPS 1 & $+12 \%,-10 \%$ & $\pm 11 \%$ \\
\hline
\end{tabular}

$\left(\beta, Q^{2}, x_{\mathbb{P}}\right)$ points. The grid points are based on the original binning scheme of the 'FPS HERA II' data. The $\left(Q^{2}, x_{\mathbb{P}}\right)$ grid points at the lowest $Q^{2}$ value of $2.5 \mathrm{GeV}^{2}$ and at the lowest and highest $x_{\mathbb{P}}$ values, which are beyond the 'FPS HERA II' data grid, are taken from the 'LPS 2' measurement.

The transformation of a measurement from the original $i$ th point $\left(\beta_{i}, Q_{i}^{2}, x_{\mathbb{P} i}\right)$ to the nearest grid point ( $\beta_{\text {grid }}, Q_{\text {grid }}^{2}$, $\left.x_{\mathbb{P} \text { grid }}\right)$ is performed by multiplying the measured cross section by the ratio $\sigma_{r}^{D(3)}\left(\beta_{\text {grid }}, Q_{\text {grid }}^{2}, x_{\mathbb{P} \text { grid }}\right) / \sigma_{r}^{D(3)}\left(\beta_{i}, Q_{i}^{2}\right.$, $x_{\mathbb{P}}$ ) calculated with the Next-to-Leading-Order (NLO) DPDF 'ZEUS SJ' parameterisation [15]. Most of the corrections are smaller than $10 \%$, while a few points undergo corrections up to $\sim 30 \%$. The procedure is checked by using the NLO DPDF 'H1 Fit B' parameterisation [5]. The resulting difference is treated as a procedural uncertainty on the combined cross section, as discussed in Sect. 2.3.2.

The cross sections from all the data sets are shown in Fig. 2 after correcting to $0.09<|t|<0.55 \mathrm{GeV}^{2}$ and transforming to the common grid.

\subsection{Combination method}

The combination is based on the $\chi^{2}$ minimisation method described in [8] and used for previous combined HERA results [10]. The averaging procedure is based on the assumption that at a given kinematic point the $\mathrm{H} 1$ and ZEUS experiments are measuring the same cross section. The correlated systematic uncertainties are floated coherently. The procedure allows a model independent check of the data consistency and leads to a significant reduction of the correlated uncertainties.

For an individual data set, the $\chi^{2}$ function is defined as:

$$
\begin{aligned}
& \chi_{\exp }^{2}(\boldsymbol{m}, \boldsymbol{b}) \\
& =\sum_{i} \frac{\left[m^{i}-\sum_{j} \gamma_{j}^{i} m^{i} b_{j}-\mu^{i}\right]^{2}}{\delta_{i, \mathrm{stat}}^{2} \mu^{i}\left(m^{i}-\sum_{j} \gamma_{j}^{i} m^{i} b_{j}\right)+\left(\delta_{i, \mathrm{uncor}} m^{i}\right)^{2}} \\
& \quad+\sum_{j} b_{j}^{2} .
\end{aligned}
$$


Fig. 2 Reduced diffractive cross section $x_{\mathbb{P}} \sigma_{r}{ }^{D(3)}\left(\beta, Q^{2}, x_{\mathbb{P}}\right)$ for $0.09<|t|<0.55 \mathrm{GeV}^{2}$ as a function of $Q^{2}$ for different values of $\beta$ and $x_{\mathbb{P}}$. The $\mathrm{H} 1$ 'FPS HERA II' [2], H1 'FPS HERA I' [1], ZEUS 'LPS 2' [4] and ZEUS 'LPS 1' [3] data are presented. The inner error bars indicate the statistical uncertainties, while the outer error bars show the statistical and systematic uncertainties added in quadrature. Normalisation uncertainties are not included in the error bars of the individual measurements

\section{H1 and ZEUS}

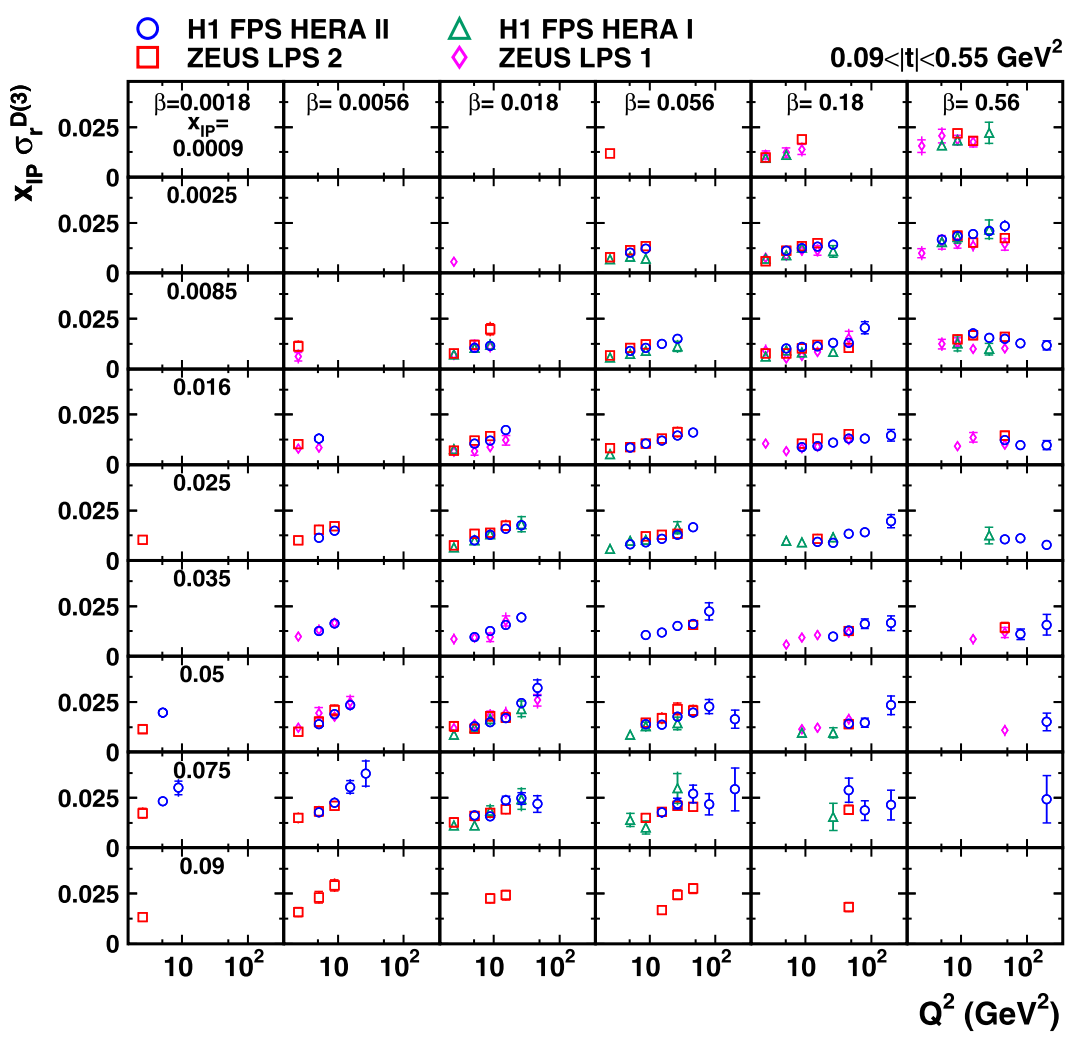

Here $\mu^{i}$ is the measured cross section value at a point $i$ $\left(\beta_{i}, Q_{i}^{2}, x_{\mathbb{P} i}\right)$, and $\gamma_{j}^{i}, \delta_{i, \text { stat }}$ and $\delta_{i, \text { uncor }}$ are the relative correlated systematic, relative statistical and relative uncorrelated systematic uncertainties, respectively. The vector $\boldsymbol{m}$ of quantities $m^{i}$ expresses the values of the combined cross section for each point $i$ and the vector $\boldsymbol{b}$ of quantities $b_{j}$ expresses the shifts of the correlated systematic uncertainty sources, $j$, in units of the standard deviation. The relative uncertainties $\gamma_{j}^{i}$ and $\delta_{i}$,uncor are multiplied by the combined cross section $m^{i}$ in order to take into account the fact that the correlated and uncorrelated systematic uncertainties are to a good approximation proportional to the central values (multiplicative uncertainties). On the other hand, the statistical uncertainties scale with the square root of the expected number of events, which is determined by the expected cross section, corrected for the biases due to the correlated systematic uncertainties. This is taken into account by the $\delta_{i, \text { stat }}^{2} \mu^{i}\left(m^{i}-\sum_{j} \gamma_{j}^{i} m^{i} b_{j}\right)$ term.

If several analyses provide measurements at the same ( $\beta$, $\left.Q^{2}, x_{\mathbb{P}}\right)$ values, a $\chi_{\mathrm{tot}}^{2}$ is built [9] from the sum of the $\chi_{\exp }^{2}$ of each data set, assuming the individual data sets to be statistically uncorrelated. The $\chi_{\text {tot }}^{2}$ is minimised with respect to the $m^{i}$ and $b_{j}$ from each data set with an iterative procedure. The ratio $\chi_{\min }^{2} / n_{\text {dof }}$ is a measure of the consistency of the data sets. The number of degrees of freedom, $n_{\mathrm{dof}}$, is calculated as the difference between the total number of measurements and the number of averaged points. The uncertainties of the combined cross sections are evaluated from the $\chi_{\min }^{2}+1$ criteria [8-10]. For some of the $\left(\beta, Q^{2}, x_{\mathbb{P}}\right)$ points there is only one measurement; however, because of the systematic uncertainty correlations such measurements may be shifted with respect to the original values, and the uncertainties may be reduced.

\subsection{Uncertainties}

\subsubsection{Experimental systematic uncertainties and their correlations}

The input cross sections are published with their statistical and systematic uncertainties. The statistical uncertainties correspond to $\delta_{i, \text { stat }}$ in Eq. (3). The systematic uncertainties are classified as point-to-point correlated or point-to-point uncorrelated, corresponding to $\gamma_{j}^{i}$ and $\delta_{i \text {,uncor }}$ respectively, according to the information provided in the corresponding publications, as follows:

- For the two older analyses, 'FPS HERA I' and 'LPS 1', only the total systematic uncertainties are given $[1,3]$, with no information on the single contributions and pointto-point correlations. For these two samples only the normalisation uncertainties (Table 2) are considered among 
the correlated systematics, while the remaining uncertainties are treated as uncorrelated.

- For the sample 'FPS HERA II' all the systematic sources discussed in [2] are treated as point-to-point correlated. The hadronic energy scale uncertainty is taken as correlated separately for $x_{\mathbb{P}}<0.012$ and $x_{\mathbb{P}}>0.012$. This is to account for the different sensitivity to this systematic source for the two $x_{\mathbb{P}}$ regions, where different methods are used to reconstruct the variable $\beta$, which are typically sensitive to different regions of the $\mathrm{H} 1$ central calorimeter. For $x_{\mathbb{P}}<0.012$, where the mass $M_{X}$ of the hadronic final state is used to reconstruct $\beta$, the effect on the cross section due to the hadronic energy scale uncertainty is $4 \%$ on average and reaches $6.7 \%$. For $x_{\mathbb{P}}>0.012$, where $\beta$ is reconstructed with the leading proton energy measured by the FPS, the cross section shows almost no sensitivity to the hadronic energy scale.

- In the 'LPS 2' case, the total systematic uncertainties quoted in [4] are decomposed in correlated and uncorrelated following the prescriptions in [15]. They are symmetrised by taking the average of the positive and negative uncertainties.

In the H1 'FPS HERA II' analysis, the systematic effects related to the leading proton measurement are considered as correlated and derived from the variation of the acceptance in the $x_{\mathbb{P}}$ and $t$ bins when shifting the FPS energy scale and transverse momentum within the estimated uncertainties [2]. In the ZEUS 'LPS 2' case, the systematic uncertainty related to the leading proton measurement is dominated by the incomplete knowledge of the beam optics, of the position of the beamline aperture limitations and of the intrinsic transverse-momentum spread of the proton beam at the interaction point. The beam optics contribution is largely independent of the kinematic variables and therefore is taken as a normalisation uncertainty [4]. The other contributions are quantified by varying the cut on the distance of closest approach of the reconstructed proton track to the beampipe, and the value of the intrinsic transverse-momentum spread assumed in the simulation. They are treated as uncorrelated uncertainties.

All the $\mathrm{H} 1$ systematic uncertainties are treated as independent of the ZEUS uncertainties, and vice versa. Possible effects due to correlations between the two experiments are taken into account in the procedural uncertainties, discussed in Sect. 2.3.2. In total, 23 independent sources of correlated systematic uncertainties are considered, including the global normalisation for each sample. The full list is given in Table 3 .
Table 3 Sources of point-to-point correlated systematic uncertainties considered in the combination. For each source the shifts resulting from the combination in units of the original uncertainty and the values of the final uncertainties as percentages of the original are given

\begin{tabular}{lcc}
\hline Source & Shift $(\sigma$ units $)$ & Reduction factor $\%$ \\
\hline FPS HERA II hadronic energy scale $x_{\mathbb{P}}<0.012$ & -1.61 & 56.9 \\
FPS HERA II hadronic energy scale $x_{\mathbb{P}}>0.012$ & 0.13 & 99.8 \\
FPS HERA II electromagnetic energy scale & 0.49 & 85.9 \\
FPS HERA II electron angle & 0.67 & 66.6 \\
FPS HERA II $\beta$ reweighting & 0.15 & 90.4 \\
FPS HERA II $x_{\mathbb{P}}$ reweighting & 0.05 & 98.3 \\
FPS HERA II $t$ reweighting & 0.70 & 79.8 \\
FPS HERA II $Q^{2}$ reweighting & 0.09 & 97.6 \\
FPS HERA II proton energy & 0.05 & 45.6 \\
FPS HERA II proton $p_{x}$ & 0.62 & 74.5 \\
FPS HERA II proton $p_{y}$ & 0.27 & 86.5 \\
FPS HERA II vertex reconstruction & 0.07 & 97.0 \\
FPS HERA II background subtraction & 0.84 & 89.9 \\
FPS HERA II bin centre corrections & -1.05 & 87.3 \\
FPS HERA II global normalisation & -0.39 & 84.4 \\
FPS HERA I global normalisation & 0.81 & 48.9 \\
LPS 2 hadronic energy scale & -0.02 & 55.0 \\
LPS 2 electromagnetic energy scale & -0.14 & 62.4 \\
LPS $2 x_{\mathbb{P}}$ reweighting & -0.32 & 98.2 \\
LPS $2 t$ reweighting & -0.26 & 86.4 \\
LPS 2 background subtraction & 0.40 & 94.9 \\
LPS 2 global normalisation & -0.53 & 67.7 \\
LPS 1 global normalisation & 0.86 & 44.1 \\
\hline
\end{tabular}




\subsubsection{Procedural uncertainties}

The following uncertainties on the combined cross sections due to the combination procedure are studied:

- The $\chi^{2}$ function given by Eq. (3) treats all systematic uncertainties as multiplicative, i.e. proportional to the expected central values. While this generally holds for the normalisation uncertainties, it may not be the case for the other uncertainties. To study the sensitivity of the average result to this issue, an alternative averaging is performed. Only the normalisation uncertainty and those related to the $t$ reconstruction (the uncertainties on the 'FPS HERA II' proton $p_{x}, p_{y}$ reconstruction and on the 'FPS HERA II' and 'LPS 2' $t$ reweighting) which, for the reasons explained in Sect. 2.1.1, can affect the normalisation, are taken as multiplicative, while all other uncertainties are treated as additive. The difference between this average and the nominal result is of the order of $1 \%$ on average and $6.4 \%$ at most.

- The H1 and ZEUS experiments use similar methods for detector calibration, apply similar reweighting to the Monte Carlo models used for the acceptance corrections and employ similar Monte Carlo models for QED radiative corrections, for the hadronic final state simulation and for background subtraction. Such similarities may lead to correlations between the measurements of the two experiments. Three systematic source are identified as the most likely to be correlated between the two experiments. These are the electromagnetic energy scale and the reweighting of the simulation in $x_{\mathbb{P}}$ and $t$. Averages are formed for each of the $2^{3}$ possible assumptions on the presence of correlations of these systematic uncertainties between the experiments and are compared with the nominal average for which all sources are assumed to be uncorrelated. The maximum difference between the nominal and the alternative averages is taken as an uncertainty. It is $1.4 \%$ on average and $6.6 \%$ at most, with no particular dependence on the kinematics.

- The bias introduced by transforming the data to the common grid (see Sect. 2.1.2) is studied by using correction factors obtained from the NLO DPDF 'H1 Fit B' [5] parameterisation. For a few bins this changes the result by up to $8 \%$, but the average effect is $1.2 \%$.

- The averaging procedure shifts the H1 hadronic energy scale at $x_{\mathbb{P}}<0.012$ by substantially more than $1 \sigma$ of the nominal value (see Sect. 3). To study the sensitivity of the average result to the treatment of the uncertainty on the $\mathrm{H} 1$ hadronic energy scale, an alternative averaging is performed for which this uncertainty is considered as pointto-point uncorrelated. The difference between the alternative and nominal results is $0.9 \%$ on average and reaches $8.7 \%$ at low $x_{\mathbb{P}}$.
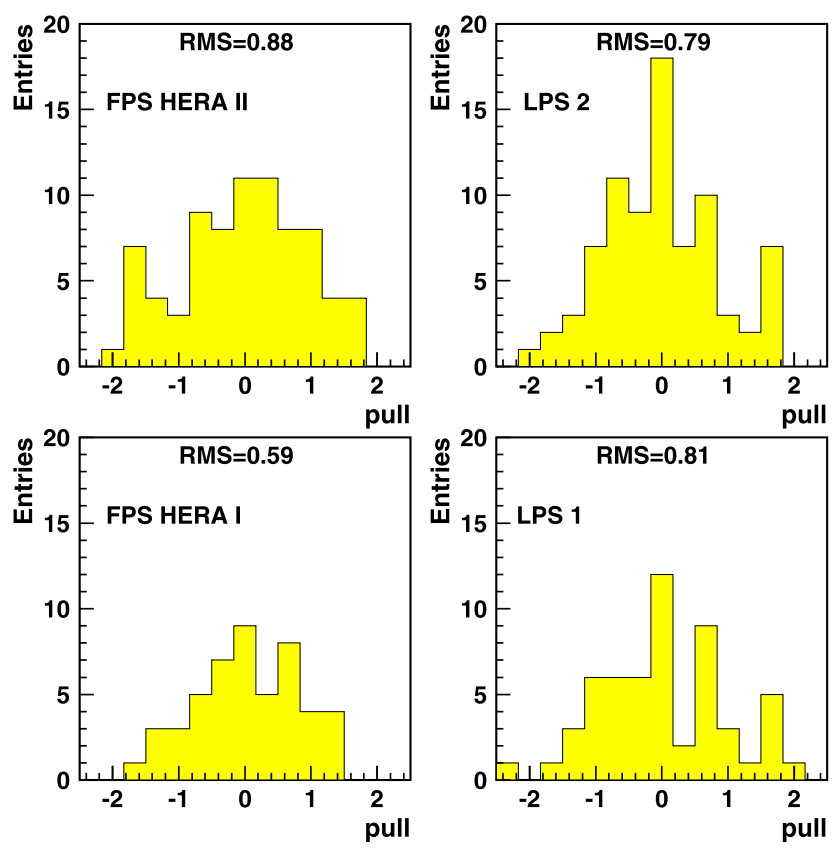

Fig. 3 Pull distributions for the individual data sets. The RMS values give the root mean square of the distributions

For each combined data point the difference between the average obtained by considering each of the procedural effects and the nominal average is calculated and summed in quadrature. The effect of the procedural uncertainties is $2.9 \%$ on average and $9.3 \%$ at most.

\section{Results}

In the minimisation procedure, 352 data points are combined to 191 cross section measurements. The data show good consistency, with $\chi_{\min }^{2} / n_{\text {dof }}=133 / 161$. The distributions of pulls [10], shown in Fig. 3 for each data set, exhibit no significant tensions. For data with no correlated systematic uncertainties pulls are expected to follow Gaussian distributions with zero mean and unit width. Correlated systematic uncertainties lead to narrowed pull distributions.

The effects of the combination on the correlated systematic uncertainties are summarised in Table 3 in terms of shifts in units of the original uncertainty and of values of the final uncertainties as percentages of the originals. The combined cross section values are given in Table 4 together with statistical, uncorrelated systematic, correlated systematic, experimental, procedural and total uncertainties. The experimental uncertainty is obtained as the quadratic sum of the statistical, uncorrelated systematic and correlated systematic uncertainties. The total uncertainty is defined as the quadratic sum of the experimental and procedural uncertainties. The full information about correlations can be found 
Table 4 Combined reduced cross sections

$x_{\mathbb{P}} \sigma_{r}{ }^{D(3)}\left(\beta, Q^{2}, x_{\mathbb{P}}\right)$ for

diffractive $e p$ scattering,

$e p \rightarrow e X p$. The values indicated

by $\delta_{\text {stat }}, \delta_{\text {uncor }}, \delta_{\text {cor }}, \delta_{\text {exp }}, \delta_{\text {proc }}$

and $\delta_{\text {tot }}$ represent the statistical,

uncorrelated systematic,

correlated systematic,

experimental, procedural and

total uncertainties, respectively

\begin{tabular}{|c|c|c|c|c|c|c|c|c|c|}
\hline $\begin{array}{l}Q^{2} \\
\left(\mathrm{GeV}^{2}\right)\end{array}$ & $\beta$ & $x_{\mathbb{P}}$ & $x_{\mathbb{P}} \sigma_{r}^{D(3)}$ & $\begin{array}{l}\delta_{\text {stat }} \\
(\%)\end{array}$ & $\begin{array}{l}\delta_{\text {uncor }} \\
(\%)\end{array}$ & $\begin{array}{l}\delta_{\text {cor }} \\
(\%)\end{array}$ & $\begin{array}{l}\delta_{\exp } \\
(\%)\end{array}$ & $\begin{array}{l}\delta_{\text {proc }} \\
(\%)\end{array}$ & $\begin{array}{l}\delta_{\text {tot }} \\
(\%)\end{array}$ \\
\hline 2.5 & 0.0018 & 0.0500 & 0.0110 & 19 & 5.8 & 4.7 & 21 & 7.6 & 22 \\
\hline 2.5 & 0.0018 & 0.0750 & 0.0166 & 14 & 6.9 & 5.3 & 17 & 7.6 & 18 \\
\hline 2.5 & 0.0018 & 0.0900 & 0.0128 & 14 & 9.6 & 5.1 & 18 & 7.9 & 20 \\
\hline 2.5 & 0.0056 & 0.0085 & 0.0101 & 19 & 11 & 7.6 & 23 & 9.3 & 25 \\
\hline 2.5 & 0.0056 & 0.0160 & 0.0093 & 12 & 6.9 & 5.1 & 14 & 3.9 & 15 \\
\hline 2.5 & 0.0056 & 0.0250 & 0.0096 & 16 & 9.8 & 5.0 & 20 & 4.6 & 20 \\
\hline 2.5 & 0.0056 & 0.0350 & 0.0110 & 18 & 11 & 4.9 & 22 & 2.3 & 22 \\
\hline 2.5 & 0.0056 & 0.0500 & 0.0117 & 9.8 & 6.4 & 5.3 & 13 & 1.5 & 13 \\
\hline 2.5 & 0.0056 & 0.0750 & 0.0143 & 14 & 11 & 5.7 & 19 & 4.7 & 19 \\
\hline 2.5 & 0.0056 & 0.0900 & 0.0154 & 15 & 6.4 & 5.7 & 17 & 4.3 & 17 \\
\hline 2.5 & 0.0178 & 0.0025 & 0.0099 & 14 & 6.8 & 4.5 & 16 & 8.2 & 18 \\
\hline 2.5 & 0.0178 & 0.0085 & 0.0076 & 8.3 & 7.1 & 4.5 & 12 & 1.7 & 12 \\
\hline 2.5 & 0.0178 & 0.0160 & 0.0073 & 8.2 & 9.5 & 4.5 & 13 & 1.4 & 13 \\
\hline 2.5 & 0.0178 & 0.0250 & 0.0071 & 8.8 & 9.2 & 4.5 & 14 & 1.4 & 14 \\
\hline 2.5 & 0.0178 & 0.0350 & 0.0095 & 15 & 29 & 4.9 & 33 & 2.3 & 33 \\
\hline 2.5 & 0.0178 & 0.0500 & 0.0114 & 7.8 & 7.1 & 4.5 & 11 & 2.2 & 12 \\
\hline 2.5 & 0.0178 & 0.0750 & 0.0123 & 11 & 7.8 & 4.9 & 14 & 1.7 & 14 \\
\hline 2.5 & 0.0562 & 0.0009 & 0.0114 & 13 & 8.6 & 5.2 & 16 & 3.4 & 17 \\
\hline 2.5 & 0.0562 & 0.0025 & 0.0074 & 9.3 & 5.7 & 4.8 & 12 & 2.8 & 12 \\
\hline 2.5 & 0.0562 & 0.0085 & 0.0064 & 9.6 & 6.7 & 4.5 & 13 & 2.3 & 13 \\
\hline 2.5 & 0.0562 & 0.0160 & 0.0068 & 10 & 10 & 4.6 & 15 & 4.4 & 16 \\
\hline 2.5 & 0.0562 & 0.0250 & 0.0063 & 14 & 14 & 4.9 & 20 & 1.9 & 20 \\
\hline 2.5 & 0.1780 & 0.0003 & 0.0156 & 8.8 & 5.4 & 4.7 & 11 & 2.6 & 12 \\
\hline 2.5 & 0.1780 & 0.0009 & 0.0102 & 5.9 & 4.3 & 4.4 & 8.5 & 2.2 & 8.8 \\
\hline 2.5 & 0.1780 & 0.0025 & 0.0068 & 8.0 & 6.3 & 4.7 & 11 & 2.6 & 12 \\
\hline 2.5 & 0.1780 & 0.0085 & 0.0074 & 9.3 & 10 & 4.8 & 15 & 3.9 & 15 \\
\hline 2.5 & 0.1780 & 0.0160 & 0.0116 & 18 & 7.5 & 5.0 & 20 & 2.3 & 20 \\
\hline 2.5 & 0.5620 & 0.0003 & 0.0214 & 16 & 8.8 & 5.0 & 19 & 2.3 & 19 \\
\hline 2.5 & 0.5620 & 0.0009 & 0.0172 & 19 & 23 & 5.0 & 31 & 2.3 & 31 \\
\hline 2.5 & 0.5620 & 0.0025 & 0.0110 & 21 & 28 & 4.9 & 36 & 2.3 & 36 \\
\hline 5.1 & 0.0018 & 0.0500 & 0.0199 & 5.9 & 0.0 & 6.6 & 8.9 & 1.8 & 9.1 \\
\hline 5.1 & 0.0018 & 0.0750 & 0.0232 & 6.7 & 0.0 & 5.1 & 8.4 & 2.1 & 8.7 \\
\hline 5.1 & 0.0056 & 0.0160 & 0.0135 & 3.9 & 0.6 & 5.9 & 7.1 & 2.0 & 7.4 \\
\hline 5.1 & 0.0056 & 0.0250 & 0.0120 & 3.4 & 0.3 & 5.2 & 6.2 & 2.0 & 6.6 \\
\hline 5.1 & 0.0056 & 0.0350 & 0.0134 & 4.0 & 0.6 & 4.7 & 6.2 & 1.5 & 6.3 \\
\hline 5.1 & 0.0056 & 0.0500 & 0.0147 & 3.9 & 0.6 & 5.4 & 6.7 & 3.4 & 7.5 \\
\hline 5.1 & 0.0056 & 0.0750 & 0.0180 & 5.7 & 1.3 & 6.1 & 8.4 & 3.7 & 9.2 \\
\hline 5.1 & 0.0056 & 0.0900 & 0.0224 & 12 & 3.8 & 4.9 & 14 & 3.1 & 14 \\
\hline 5.1 & 0.0178 & 0.0085 & 0.0120 & 2.6 & 0.4 & 5.9 & 6.4 & 7.6 & 10 \\
\hline 5.1 & 0.0178 & 0.0160 & 0.0111 & 2.6 & 0.2 & 5.2 & 5.8 & 2.8 & 6.5 \\
\hline 5.1 & 0.0178 & 0.0250 & 0.0109 & 3.0 & 0.5 & 5.2 & 6.0 & 2.2 & 6.4 \\
\hline 5.1 & 0.0178 & 0.0350 & 0.0101 & 4.3 & 0.6 & 5.2 & 6.8 & 2.2 & 7.2 \\
\hline 5.1 & 0.0178 & 0.0500 & 0.0134 & 4.1 & 1.4 & 5.1 & 6.7 & 2.2 & 7.0 \\
\hline 5.1 & 0.0178 & 0.0750 & 0.0154 & 6.4 & 2.2 & 4.8 & 8.3 & 2.9 & 8.8 \\
\hline 5.1 & 0.0562 & 0.0025 & 0.0107 & 2.4 & 0.2 & 5.0 & 5.6 & 3.4 & 6.8 \\
\hline 5.1 & 0.0562 & 0.0085 & 0.0088 & 2.7 & 0.3 & 5.0 & 5.7 & 3.5 & 6.7 \\
\hline 5.1 & 0.0562 & 0.0160 & 0.0088 & 3.2 & 0.3 & 5.1 & 6.0 & 2.7 & 6.6 \\
\hline 5.1 & 0.0562 & 0.0250 & 0.0084 & 4.5 & 0.7 & 5.0 & 6.7 & 3.1 & 7.4 \\
\hline
\end{tabular}


Table 4 (Continued)

\begin{tabular}{|c|c|c|c|c|c|c|c|c|c|}
\hline $\begin{array}{l}Q^{2} \\
\left(\mathrm{GeV}^{2}\right)\end{array}$ & $\beta$ & $x_{\mathbb{P}}$ & $x_{\mathbb{P}} \sigma_{r}^{D(3)}$ & $\begin{array}{l}\delta_{\text {stat }} \\
(\%)\end{array}$ & $\begin{array}{l}\delta_{\text {uncor }} \\
(\%)\end{array}$ & $\begin{array}{l}\delta_{\text {cor }} \\
(\%)\end{array}$ & $\begin{array}{l}\delta_{\exp } \\
(\%)\end{array}$ & $\begin{array}{l}\delta_{\text {proc }} \\
(\%)\end{array}$ & $\begin{array}{l}\delta_{\text {tot }} \\
(\%)\end{array}$ \\
\hline 5.1 & 0.0562 & 0.0500 & 0.0095 & 16 & 13 & 4.9 & 21 & 1.9 & 21 \\
\hline 5.1 & 0.0562 & 0.0750 & 0.0153 & 23 & 14 & 5.0 & 27 & 1.9 & 27 \\
\hline 5.1 & 0.1780 & 0.0009 & 0.0121 & 11 & 7.4 & 4.9 & 14 & 11 & 18 \\
\hline 5.1 & 0.1780 & 0.0025 & 0.0118 & 1.6 & 0.2 & 5.9 & 6.1 & 4.2 & 7.4 \\
\hline 5.1 & 0.1780 & 0.0085 & 0.0095 & 2.8 & 0.5 & 5.0 & 5.8 & 3.5 & 6.7 \\
\hline 5.1 & 0.1780 & 0.0160 & 0.0075 & 14 & 12 & 4.9 & 19 & 2.3 & 19 \\
\hline 5.1 & 0.1780 & 0.0250 & 0.0107 & 13 & 13 & 4.9 & 20 & 1.9 & 20 \\
\hline 5.1 & 0.1780 & 0.0350 & 0.0065 & 20 & 14 & 5.0 & 25 & 2.3 & 25 \\
\hline 5.1 & 0.5620 & 0.0003 & 0.0275 & 13 & 8.2 & 4.9 & 16 & 2.3 & 16 \\
\hline 5.1 & 0.5620 & 0.0009 & 0.0187 & 7.0 & 8.0 & 4.6 & 12 & 1.8 & 12 \\
\hline 5.1 & 0.5620 & 0.0025 & 0.0153 & 1.4 & 0.1 & 6.1 & 6.2 & 6.1 & 8.7 \\
\hline 5.1 & 0.5620 & 0.0085 & 0.0137 & 19 & 19 & 4.9 & 27 & 2.3 & 27 \\
\hline 8.8 & 0.0018 & 0.0750 & 0.0288 & 12 & 0.0 & 6.2 & 13 & 1.5 & 13 \\
\hline 8.8 & 0.0056 & 0.0250 & 0.0152 & 5.0 & 0.8 & 5.1 & 7.2 & 2.0 & 7.5 \\
\hline 8.8 & 0.0056 & 0.0350 & 0.0171 & 5.1 & 1.2 & 4.9 & 7.2 & 1.7 & 7.4 \\
\hline 8.8 & 0.0056 & 0.0500 & 0.0197 & 4.1 & 1.2 & 4.6 & 6.3 & 1.6 & 6.5 \\
\hline 8.8 & 0.0056 & 0.0750 & 0.0212 & 5.9 & 1.1 & 4.8 & 7.7 & 3.8 & 8.6 \\
\hline 8.8 & 0.0056 & 0.0900 & 0.0281 & 9.6 & 4.4 & 5.0 & 12 & 5.7 & 13 \\
\hline 8.8 & 0.0178 & 0.0085 & 0.0128 & 4.2 & 0.9 & 5.1 & 6.7 & 4.0 & 7.8 \\
\hline 8.8 & 0.0178 & 0.0160 & 0.0124 & 3.1 & 0.6 & 4.9 & 5.8 & 1.5 & 6.0 \\
\hline 8.8 & 0.0178 & 0.0250 & 0.0133 & 3.4 & 0.6 & 4.8 & 5.9 & 1.5 & 6.1 \\
\hline 8.8 & 0.0178 & 0.0350 & 0.0130 & 4.5 & 0.5 & 4.8 & 6.6 & 1.4 & 6.8 \\
\hline 8.8 & 0.0178 & 0.0500 & 0.0159 & 3.8 & 1.0 & 4.6 & 6.1 & 1.5 & 6.3 \\
\hline 8.8 & 0.0178 & 0.0750 & 0.0162 & 5.6 & 1.7 & 4.8 & 7.6 & 2.3 & 8.0 \\
\hline 8.8 & 0.0178 & 0.0900 & 0.0220 & 9.5 & 5.9 & 5.0 & 12 & 2.7 & 13 \\
\hline 8.8 & 0.0562 & 0.0025 & 0.0125 & 3.4 & 0.4 & 5.0 & 6.1 & 3.8 & 7.1 \\
\hline 8.8 & 0.0562 & 0.0085 & 0.0106 & 3.2 & 0.6 & 5.0 & 6.0 & 2.0 & 6.3 \\
\hline 8.8 & 0.0562 & 0.0160 & 0.0108 & 2.9 & 0.2 & 5.0 & 5.8 & 2.7 & 6.4 \\
\hline 8.8 & 0.0562 & 0.0250 & 0.0098 & 3.6 & 0.5 & 5.0 & 6.2 & 2.5 & 6.7 \\
\hline 8.8 & 0.0562 & 0.0350 & 0.0109 & 5.2 & 0.0 & 4.9 & 7.2 & 2.1 & 7.5 \\
\hline 8.8 & 0.0562 & 0.0500 & 0.0144 & 5.1 & 1.1 & 5.1 & 7.3 & 2.4 & 7.7 \\
\hline 8.8 & 0.0562 & 0.0750 & 0.0140 & 11 & 4.3 & 4.6 & 12 & 1.7 & 13 \\
\hline 8.8 & 0.1780 & 0.0009 & 0.0177 & 7.7 & 2.7 & 5.0 & 9.6 & 1.6 & 9.7 \\
\hline 8.8 & 0.1780 & 0.0025 & 0.0129 & 2.3 & 0.4 & 5.1 & 5.6 & 2.5 & 6.1 \\
\hline 8.8 & 0.1780 & 0.0085 & 0.0104 & 2.6 & 0.4 & 4.6 & 5.3 & 2.7 & 5.9 \\
\hline 8.8 & 0.1780 & 0.0160 & 0.0090 & 3.9 & 0.7 & 5.3 & 6.6 & 2.6 & 7.1 \\
\hline 8.8 & 0.1780 & 0.0250 & 0.0098 & 14 & 14 & 4.9 & 21 & 1.9 & 21 \\
\hline 8.8 & 0.1780 & 0.0350 & 0.0103 & 17 & 11 & 4.9 & 21 & 2.3 & 21 \\
\hline 8.8 & 0.1780 & 0.0500 & 0.0116 & 12 & 8.3 & 4.5 & 15 & 1.8 & 16 \\
\hline 8.8 & 0.5620 & 0.0003 & 0.0250 & 7.1 & 4.2 & 4.4 & 9.3 & 8.9 & 13 \\
\hline 8.8 & 0.5620 & 0.0009 & 0.0207 & 5.6 & 3.5 & 4.4 & 7.9 & 6.7 & 10 \\
\hline 8.8 & 0.5620 & 0.0025 & 0.0166 & 1.6 & 0.1 & 6.1 & 6.3 & 8.3 & 10 \\
\hline 8.8 & 0.5620 & 0.0085 & 0.0142 & 8.5 & 4.3 & 4.3 & 10 & 8.0 & 13 \\
\hline 8.8 & 0.5620 & 0.0160 & 0.0102 & 17 & 13 & 4.4 & 22 & 2.3 & 22 \\
\hline 15.3 & 0.0056 & 0.0500 & 0.0245 & 6.7 & 2.2 & 4.9 & 8.6 & 1.1 & 8.7 \\
\hline 15.3 & 0.0056 & 0.0750 & 0.0296 & 10 & 0.0 & 5.7 & 12 & 1.6 & 12 \\
\hline 15.3 & 0.0178 & 0.0160 & 0.0176 & 4.8 & 0.7 & 5.0 & 7.0 & 2.4 & 7.4 \\
\hline 15.3 & 0.0178 & 0.0250 & 0.0164 & 4.4 & 0.7 & 4.8 & 6.6 & 2.4 & 7.0 \\
\hline
\end{tabular}


Table 4 (Continued)

\begin{tabular}{|c|c|c|c|c|c|c|c|c|c|}
\hline $\begin{array}{l}Q^{2} \\
\left(\mathrm{GeV}^{2}\right)\end{array}$ & $\beta$ & $x_{\mathbb{P}}$ & $x_{\mathbb{P}} \sigma_{r}^{D(3)}$ & $\begin{array}{l}\delta_{\text {stat }} \\
(\%)\end{array}$ & $\begin{array}{l}\delta_{\text {uncor }} \\
(\%)\end{array}$ & $\begin{array}{l}\delta_{\text {cor }} \\
(\%)\end{array}$ & $\begin{array}{l}\delta_{\exp } \\
(\%)\end{array}$ & $\begin{array}{l}\delta_{\text {proc }} \\
(\%)\end{array}$ & $\begin{array}{l}\delta_{\text {tot }} \\
(\%)\end{array}$ \\
\hline 15.3 & 0.0178 & 0.0350 & 0.0165 & 5.7 & 1.1 & 4.7 & 7.5 & 1.4 & 7.6 \\
\hline 15.3 & 0.0178 & 0.0500 & 0.0176 & 4.9 & 1.4 & 4.8 & 7.0 & 2.2 & 7.4 \\
\hline 15.3 & 0.0178 & 0.0750 & 0.0211 & 6.7 & 2.1 & 4.8 & 8.5 & 2.6 & 8.9 \\
\hline 15.3 & 0.0178 & 0.0900 & 0.0234 & 10 & 1.6 & 4.8 & 11 & 3.3 & 12 \\
\hline 15.3 & 0.0562 & 0.0085 & 0.0134 & 4.5 & 0.0 & 6.0 & 7.5 & 6.1 & 9.7 \\
\hline 15.3 & 0.0562 & 0.0160 & 0.0122 & 3.9 & 0.3 & 4.9 & 6.3 & 2.5 & 6.8 \\
\hline 15.3 & 0.0562 & 0.0250 & 0.0113 & 4.5 & 0.3 & 4.8 & 6.6 & 1.0 & 6.7 \\
\hline 15.3 & 0.0562 & 0.0350 & 0.0121 & 6.2 & 0.0 & 5.0 & 8.0 & 2.0 & 8.2 \\
\hline 15.3 & 0.0562 & 0.0500 & 0.0140 & 5.7 & 1.1 & 4.9 & 7.6 & 2.0 & 7.8 \\
\hline 15.3 & 0.0562 & 0.0750 & 0.0174 & 7.6 & 1.4 & 4.7 & 9.1 & 2.1 & 9.3 \\
\hline 15.3 & 0.0562 & 0.0900 & 0.0162 & 10 & 3.6 & 5.1 & 12 & 2.8 & 12 \\
\hline 15.3 & 0.1780 & 0.0025 & 0.0136 & 3.4 & 0.5 & 5.0 & 6.0 & 1.3 & 6.2 \\
\hline 15.3 & 0.1780 & 0.0085 & 0.0111 & 3.4 & 0.5 & 4.8 & 5.9 & 2.2 & 6.2 \\
\hline 15.3 & 0.1780 & 0.0160 & 0.0098 & 3.9 & 0.6 & 5.0 & 6.4 & 2.2 & 6.8 \\
\hline 15.3 & 0.1780 & 0.0250 & 0.0097 & 6.1 & 0.9 & 5.2 & 8.1 & 2.4 & 8.4 \\
\hline 15.3 & 0.1780 & 0.0350 & 0.0117 & 15 & 17 & 4.9 & 23 & 2.3 & 23 \\
\hline 15.3 & 0.1780 & 0.0500 & 0.0134 & 12 & 15 & 4.9 & 20 & 2.3 & 20 \\
\hline 15.3 & 0.5620 & 0.0009 & 0.0180 & 8.8 & 3.4 & 4.6 & 11 & 3.3 & 11 \\
\hline 15.3 & 0.5620 & 0.0025 & 0.0173 & 2.5 & 0.2 & 5.8 & 6.3 & 3.5 & 7.2 \\
\hline 15.3 & 0.5620 & 0.0085 & 0.0162 & 3.3 & 0.5 & 5.1 & 6.1 & 3.0 & 6.8 \\
\hline 15.3 & 0.5620 & 0.0160 & 0.0151 & 17 & 14 & 4.9 & 22 & 2.3 & 22 \\
\hline 15.3 & 0.5620 & 0.0350 & 0.0094 & 20 & 21 & 4.9 & 30 & 2.3 & 30 \\
\hline 26.5 & 0.0056 & 0.0750 & 0.0359 & 17 & 0.0 & 5.3 & 18 & 3.2 & 18 \\
\hline 26.5 & 0.0178 & 0.0250 & 0.0179 & 8.0 & 1.4 & 4.8 & 9.4 & 2.3 & 9.7 \\
\hline 26.5 & 0.0178 & 0.0350 & 0.0202 & 8.6 & 0.0 & 5.3 & 10 & 1.6 & 10 \\
\hline 26.5 & 0.0178 & 0.0500 & 0.0250 & 6.7 & 1.3 & 4.8 & 8.4 & 1.8 & 8.6 \\
\hline 26.5 & 0.0178 & 0.0750 & 0.0249 & 10 & 2.3 & 5.2 & 12 & 2.6 & 12 \\
\hline 26.5 & 0.0562 & 0.0085 & 0.0157 & 6.6 & 1.2 & 5.3 & 8.6 & 8.0 & 12 \\
\hline 26.5 & 0.0562 & 0.0160 & 0.0150 & 4.9 & 0.7 & 4.8 & 7.0 & 1.8 & 7.2 \\
\hline 26.5 & 0.0562 & 0.0250 & 0.0134 & 5.5 & 0.7 & 4.5 & 7.1 & 1.3 & 7.3 \\
\hline 26.5 & 0.0562 & 0.0350 & 0.0157 & 7.4 & 0.0 & 4.8 & 8.8 & 1.6 & 9.0 \\
\hline 26.5 & 0.0562 & 0.0500 & 0.0184 & 6.2 & 1.6 & 5.1 & 8.2 & 1.3 & 8.3 \\
\hline 26.5 & 0.0562 & 0.0750 & 0.0211 & 7.4 & 1.8 & 4.5 & 8.9 & 1.5 & 9.0 \\
\hline 26.5 & 0.0562 & 0.0900 & 0.0237 & 9.6 & 3.2 & 5.0 & 11 & 3.4 & 12 \\
\hline 26.5 & 0.1780 & 0.0025 & 0.0138 & 5.4 & 0.4 & 5.1 & 7.5 & 1.4 & 7.6 \\
\hline 26.5 & 0.1780 & 0.0085 & 0.0126 & 5.0 & 0.8 & 4.8 & 7.0 & 2.7 & 7.5 \\
\hline 26.5 & 0.1780 & 0.0160 & 0.0113 & 5.5 & 0.0 & 5.1 & 7.6 & 2.2 & 7.9 \\
\hline 26.5 & 0.1780 & 0.0250 & 0.0093 & 6.5 & 1.0 & 4.9 & 8.2 & 1.4 & 8.3 \\
\hline 26.5 & 0.1780 & 0.0350 & 0.0100 & 9.8 & 0.0 & 5.7 & 11 & 4.0 & 12 \\
\hline 26.5 & 0.1780 & 0.0500 & 0.0105 & 26 & 14 & 4.9 & 30 & 1.9 & 30 \\
\hline 26.5 & 0.1780 & 0.0750 & 0.0169 & 42 & 11 & 4.9 & 44 & 1.9 & 44 \\
\hline 26.5 & 0.5620 & 0.0009 & 0.0241 & 22 & 10 & 4.9 & 25 & 1.9 & 25 \\
\hline 26.5 & 0.5620 & 0.0025 & 0.0189 & 3.7 & 0.2 & 6.0 & 7.0 & 9.1 & 12 \\
\hline 26.5 & 0.5620 & 0.0085 & 0.0140 & 4.3 & 0.4 & 5.0 & 6.6 & 3.8 & 7.6 \\
\hline 26.5 & 0.5620 & 0.0250 & 0.0136 & 31 & 15 & 4.9 & 35 & 1.9 & 35 \\
\hline 46 & 0.0178 & 0.0500 & 0.0313 & 8.6 & 4.5 & 4.7 & 11 & 1.6 & 11 \\
\hline 46 & 0.0178 & 0.0750 & 0.0218 & 19 & 0.0 & 5.1 & 20 & 2.5 & 20 \\
\hline 46 & 0.0562 & 0.0160 & 0.0163 & 8.8 & 0.0 & 5.2 & 10 & 2.1 & 11 \\
\hline
\end{tabular}


Table 4 (Continued)

\begin{tabular}{|c|c|c|c|c|c|c|c|c|c|}
\hline $\begin{array}{l}Q^{2} \\
\left(\mathrm{GeV}^{2}\right)\end{array}$ & $\beta$ & $x_{\mathbb{P}}$ & $x_{\mathbb{P}} \sigma_{r}^{D(3)}$ & $\begin{array}{l}\delta_{\text {stat }} \\
(\%)\end{array}$ & $\begin{array}{l}\delta_{\text {uncor }} \\
(\%)\end{array}$ & $\begin{array}{l}\delta_{\text {cor }} \\
(\%)\end{array}$ & $\begin{array}{l}\delta_{\exp } \\
(\%)\end{array}$ & $\begin{array}{l}\delta_{\text {proc }} \\
(\%)\end{array}$ & $\begin{array}{l}\delta_{\text {tot }} \\
(\%)\end{array}$ \\
\hline 46 & 0.0562 & 0.0250 & 0.0172 & 8.6 & 0.0 & 5.3 & 10 & 2.1 & 10 \\
\hline 46 & 0.0562 & 0.0350 & 0.0158 & 8.3 & 1.8 & 4.6 & 9.6 & 2.2 & 9.8 \\
\hline 46 & 0.0562 & 0.0500 & 0.0199 & 7.6 & 1.9 & 4.8 & 9.2 & 2.8 & 9.6 \\
\hline 46 & 0.0562 & 0.0750 & 0.0212 & 8.4 & 1.2 & 4.9 & 9.7 & 3.2 & 10 \\
\hline 46 & 0.0562 & 0.0900 & 0.0267 & 8.9 & 2.4 & 4.8 & 10 & 1.0 & 10 \\
\hline 46 & 0.1780 & 0.0085 & 0.0121 & 6.6 & 1.3 & 5.4 & 8.6 & 2.1 & 8.9 \\
\hline 46 & 0.1780 & 0.0160 & 0.0133 & 5.9 & 1.5 & 4.8 & 7.7 & 2.4 & 8.1 \\
\hline 46 & 0.1780 & 0.0250 & 0.0135 & 8.5 & 0.0 & 4.9 & 9.8 & 2.2 & 10 \\
\hline 46 & 0.1780 & 0.0350 & 0.0129 & 7.5 & 1.9 & 4.6 & 9.0 & 2.1 & 9.2 \\
\hline 46 & 0.1780 & 0.0500 & 0.0148 & 7.4 & 2.9 & 4.8 & 9.3 & 2.4 & 9.6 \\
\hline 46 & 0.1780 & 0.0750 & 0.0201 & 9.9 & 4.0 & 4.7 & 12 & 3.4 & 12 \\
\hline 46 & 0.1780 & 0.0900 & 0.0177 & 13 & 4.2 & 5.0 & 14 & 8.6 & 17 \\
\hline 46 & 0.5620 & 0.0025 & 0.0196 & 5.1 & 1.0 & 5.4 & 7.5 & 4.2 & 8.6 \\
\hline 46 & 0.5620 & 0.0085 & 0.0135 & 5.1 & 1.0 & 4.9 & 7.2 & 4.6 & 8.5 \\
\hline 46 & 0.5620 & 0.0160 & 0.0124 & 6.9 & 1.8 & 4.8 & 8.6 & 2.3 & 8.9 \\
\hline 46 & 0.5620 & 0.0250 & 0.0106 & 13 & 0.0 & 5.9 & 14 & 1.2 & 15 \\
\hline 46 & 0.5620 & 0.0350 & 0.0135 & 14 & 7.0 & 4.8 & 16 & 2.2 & 16 \\
\hline 46 & 0.5620 & 0.0500 & 0.0120 & 17 & 20 & 4.9 & 26 & 2.3 & 26 \\
\hline 46 & 0.8160 & 0.0009 & 0.0145 & 21 & 5.3 & 4.5 & 22 & 1.4 & 22 \\
\hline 46 & 0.8160 & 0.0025 & 0.0131 & 17 & 8.1 & 5.3 & 20 & 3.0 & 20 \\
\hline 46 & 0.8160 & 0.0085 & 0.0110 & 18 & 3.9 & 4.3 & 19 & 1.5 & 19 \\
\hline 46 & 0.8160 & 0.0160 & 0.0092 & 27 & 3.9 & 5.4 & 28 & 4.1 & 28 \\
\hline 80 & 0.0562 & 0.0350 & 0.0227 & 19 & 0.0 & 5.8 & 20 & 2.7 & 20 \\
\hline 80 & 0.0562 & 0.0500 & 0.0235 & 15 & 0.0 & 5.0 & 16 & 2.0 & 16 \\
\hline 80 & 0.0562 & 0.0750 & 0.0216 & 24 & 0.0 & 5.9 & 25 & 1.9 & 25 \\
\hline 80 & 0.1780 & 0.0085 & 0.0206 & 15 & 0.0 & 6.0 & 16 & 2.9 & 16 \\
\hline 80 & 0.1780 & 0.0160 & 0.0133 & 13 & 0.0 & 4.8 & 14 & 2.3 & 14 \\
\hline 80 & 0.1780 & 0.0250 & 0.0146 & 12 & 0.0 & 5.2 & 13 & 1.6 & 13 \\
\hline 80 & 0.1780 & 0.0350 & 0.0162 & 14 & 0.0 & 5.6 & 15 & 1.0 & 15 \\
\hline 80 & 0.1780 & 0.0500 & 0.0146 & 15 & 0.0 & 5.5 & 16 & 2.3 & 16 \\
\hline 80 & 0.1780 & 0.0750 & 0.0183 & 26 & 0.0 & 5.3 & 27 & 3.0 & 27 \\
\hline 80 & 0.5620 & 0.0085 & 0.0116 & 10 & 0.0 & 6.4 & 12 & 5.1 & 13 \\
\hline 80 & 0.5620 & 0.0160 & 0.0090 & 14 & 0.0 & 7.0 & 15 & 3.5 & 16 \\
\hline 80 & 0.5620 & 0.0250 & 0.0104 & 17 & 0.0 & 6.7 & 18 & 5.3 & 19 \\
\hline 80 & 0.5620 & 0.0350 & 0.0109 & 25 & 0.0 & 7.3 & 26 & 3.6 & 26 \\
\hline 200 & 0.0562 & 0.0500 & 0.0162 & 28 & 0.0 & 5.0 & 28 & 1.0 & 28 \\
\hline 200 & 0.0562 & 0.0750 & 0.0288 & 37 & 0.0 & 5.5 & 37 & 2.3 & 37 \\
\hline 200 & 0.1780 & 0.0160 & 0.0145 & 20 & 0.0 & 5.8 & 21 & 1.3 & 21 \\
\hline 200 & 0.1780 & 0.0250 & 0.0199 & 16 & 0.0 & 5.0 & 17 & 1.9 & 17 \\
\hline 200 & 0.1780 & 0.0350 & 0.0169 & 22 & 0.0 & 5.2 & 23 & 2.6 & 23 \\
\hline 200 & 0.1780 & 0.0500 & 0.0235 & 20 & 0.0 & 5.5 & 21 & 2.6 & 21 \\
\hline 200 & 0.1780 & 0.0750 & 0.0209 & 35 & 0.0 & 5.6 & 35 & 2.5 & 36 \\
\hline 200 & 0.5620 & 0.0085 & 0.0109 & 19 & 0.0 & 6.6 & 21 & 3.9 & 21 \\
\hline 200 & 0.5620 & 0.0160 & 0.0093 & 23 & 0.0 & 6.4 & 24 & 1.9 & 24 \\
\hline 200 & 0.5620 & 0.0250 & 0.0074 & 27 & 0.0 & 6.7 & 28 & 4.9 & 29 \\
\hline 200 & 0.5620 & 0.0350 & 0.0158 & 33 & 0.0 & 6.7 & 34 & 2.4 & 34 \\
\hline 200 & 0.5620 & 0.0500 & 0.0151 & 29 & 0.0 & 5.4 & 29 & 1.8 & 29 \\
\hline 200 & 0.5620 & 0.0750 & 0.0228 & 50 & 0.0 & 5.9 & 50 & 3.2 & 50 \\
\hline
\end{tabular}


elsewhere. ${ }^{3}$ As the global normalisations of the input data sets are fitted as correlated systematic uncertainties, the normalisation uncertainty on the combined data is included in the correlated systematic uncertainty given in Table 4 .

Most of the 23 correlated systematic uncertainties shift by less than $0.5 \sigma$ of the nominal value in the averaging procedure. None of them shifts by substantially more than $1 \sigma$, with the exception of the hadronic energy scale for $x_{\mathbb{P}}<0.012$ for the 'FPS HERA II' sample. Detailed studies show that there is a tension between the H1 'FPS HERA II' and ZEUS 'LPS 2' data at low $x_{\mathbb{P}}$; the average ratio of the H1 to ZEUS cross sections is above 1.0 for $\beta>0.1$ and below 0.9 for $\beta<0.1$. The H1 cross section uncertainty is positively correlated with the hadronic energy scale for $\beta>0.1$ and anti-correlated for $\beta<0.1$. As a result, the combination shifts the $\mathrm{H} 1$ cross sections for $x_{\mathbb{P}}<0.012$ in the direction opposite to the cross section uncertainty due to the $\mathrm{H} 1$ hadronic energy scale. Conversely the combined statistical and uncorrelated uncertainty on the ZEUS data is much larger than the ZEUS hadronic energy scale uncertainty; consequently the fit is less sensitive to the ZEUS hadronic energy scale.

The influence of several correlated systematic uncertainties is reduced significantly for the combined result. Specifically, the uncertainty on the FPS proton energy measurement and the normalisation uncertainties on the 'FPS HERA I' and 'LPS 1' samples are reduced by more than a factor of 2. The $\mathrm{H} 1$ hadronic energy scale uncertainty for the low $x_{\mathbb{P}}$-range $\left(x_{\mathbb{P}}<0.012\right)$ and the ZEUS hadronic energy scale uncertainty are reduced to around $55 \%$ of those for the individual data sets. Since H1 and ZEUS use different reconstruction methods, similar systematic sources influence the measured cross section differently. Therefore, requiring the cross sections to be consistent at all $\left(\beta, Q^{2}, x_{\mathbb{P}}\right)$ points constrains the systematic uncertainties efficiently. Due to this cross calibration effect, the combined measurement shows an average improvement of the experimental uncertainty of about $27 \%$ with respect to the most precise single data set, 'FPS HERA II', though the latter data set contains five times more events than the second largest data set, 'LPS 2'. The correlated part of the experimental uncertainty is reduced from about $69 \%$ in [2] to $49 \%$ in the combined measurement. The statistical, experimental and procedural uncertainties on the combined data are on average $11 \%, 13.8 \%$ and $2.9 \%$, respectively. The total uncertainty on the cross section is $14.3 \%$ on average and is $6 \%$ for the most precise points. The normalisation uncertainty, which contributes to the correlated systematic uncertainty on the combined data, is on average $4 \%$. The combined result extends the kinematic coverage with respect to the H1 and ZEUS measurements taken separately and the resulting cross section covers

\footnotetext{
${ }^{3}$ The combined data together with the full correlation information are provided at the URL. http://www.desy.de/h1zeus
}

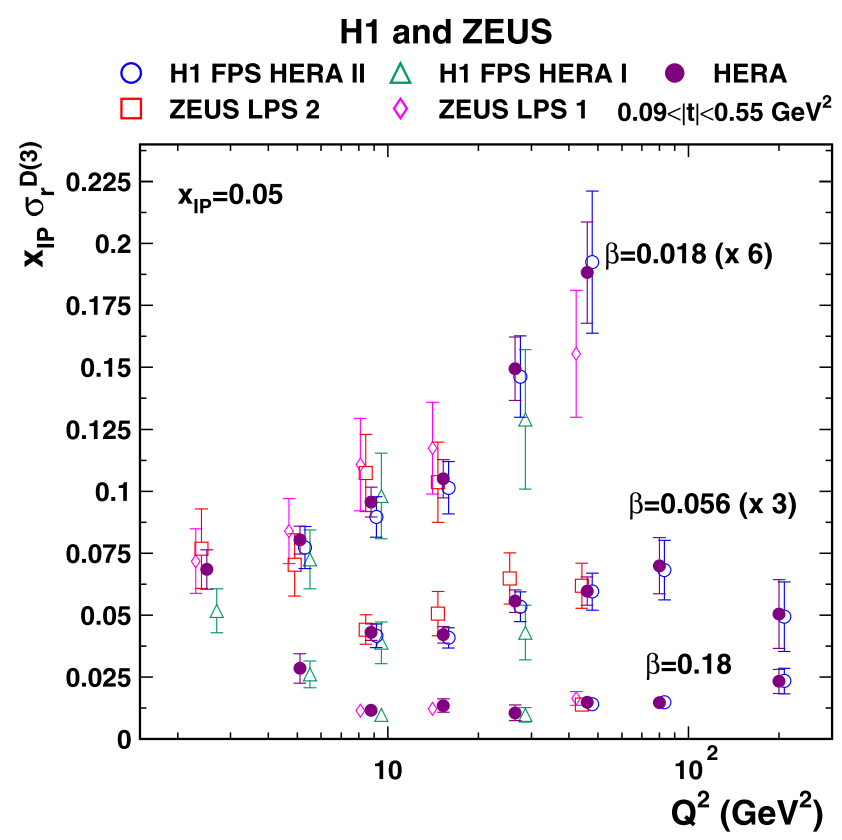

Fig. 4 Reduced diffractive cross section $x_{\mathbb{P}} \sigma_{r}^{D(3)}\left(\beta, Q^{2}, x_{\mathbb{P}}\right)$ for $0.09<|t|<0.55 \mathrm{GeV}^{2}$ as a function of $Q^{2}$ for different values of $\beta$ at $x_{\mathbb{P}}=0.05$. The HERA combined data are compared to the $\mathrm{H} 1$ and ZEUS data inputs to the averaging procedure. The error bars indicate the statistical and systematic uncertainties added in quadrature for the input measurements and the statistical, systematic and procedural uncertainties added in quadrature for the combined points. Normalisation uncertainties are not included in the error bars of the individual measurements, whereas they are included in the error bars of the combined points

the region $2.5 \leq Q^{2} \leq 200 \mathrm{GeV}^{2}, 0.0018 \leq \beta \leq 0.816$ and $0.00035 \leq x_{\mathbb{P}} \leq 0.09$, for $0.09<|t|<0.55 \mathrm{GeV}^{2}$. Figure 4 shows the HERA combined cross section as a function of $Q^{2}$ at $x_{\mathbb{P}}=0.05$, for different values of $\beta$, compared with the individual measurements used for the combination. The reduction of the total uncertainty of the HERA measurement compared to the input cross sections is visible. The derivative of the reduced cross section as a function of $\log \left(Q^{2}\right)$ decreases with $\beta$, a feature characteristic of the scaling violations in diffractive DIS, which are now measured precisely from proton-tagged as well as LRG data. Figures 5 and 6 show the HERA combined diffractive reduced cross sections as a function of $Q^{2}$ and $x_{\mathbb{P}}$, respectively.

At low $x_{\mathbb{P}} \lesssim 0.01$, where the proton spectrometer data are free from proton dissociation contributions, the combined data provide the most precise determination of the absolute normalisation of the diffractive cross section.

\section{Conclusions}

The reduced diffractive cross sections, $\sigma_{r}{ }^{D(3)}(e p \rightarrow e X p)$, measured by the $\mathrm{H} 1$ and ZEUS Collaborations by using proton spectrometers to detect the leading protons are com- 
Fig. 5 HERA combined reduced diffractive cross section $x_{\mathbb{P}} \sigma_{r}{ }^{D(3)}\left(\beta, Q^{2}, x_{\mathbb{P}}\right)$ for $0.09<|t|<0.55 \mathrm{GeV}^{2}$ as a function of $Q^{2}$ for different values of $\beta$ and $x_{\mathbb{P}}$. The error bars indicate the statistical, systematic and procedural uncertainties added in quadrature. The normalisation uncertainty is included

Fig. 6 HERA combined reduced diffractive cross section $x_{\mathbb{P}} \sigma_{r}^{D(3)}\left(\beta, Q^{2}, x_{\mathbb{P}}\right)$ for $0.09<|t|<0.55 \mathrm{GeV}^{2}$ as a function of $x_{\mathbb{P}}$ for different values of $\beta$ and $Q^{2}$. The error bars indicate the statistical, systematic and procedural uncertainties added in quadrature. The normalisation uncertainty is included
H1 and ZEUS

HERA

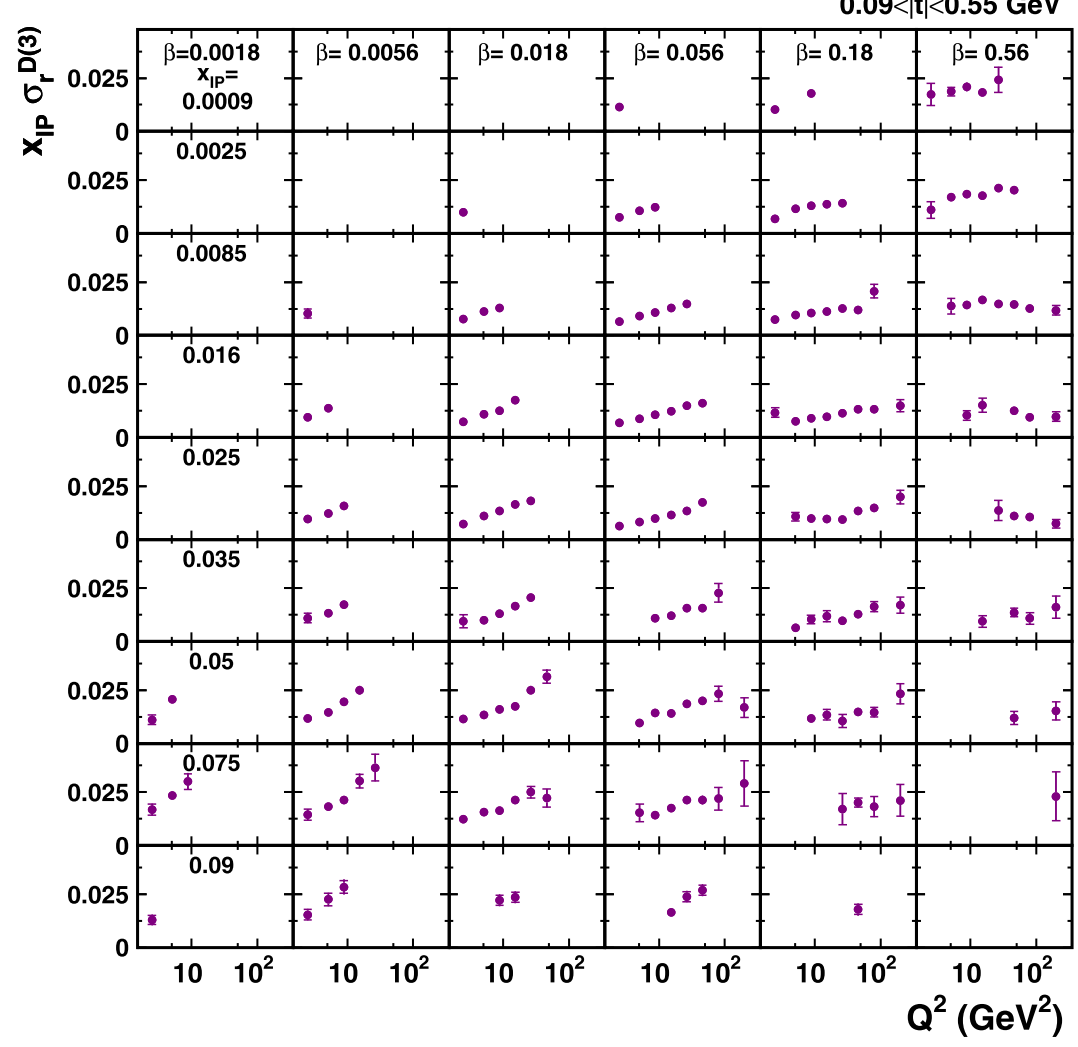

H1 and ZEUS

$0.09<|\mathrm{t}|<0.55 \mathrm{GeV}^{2}$

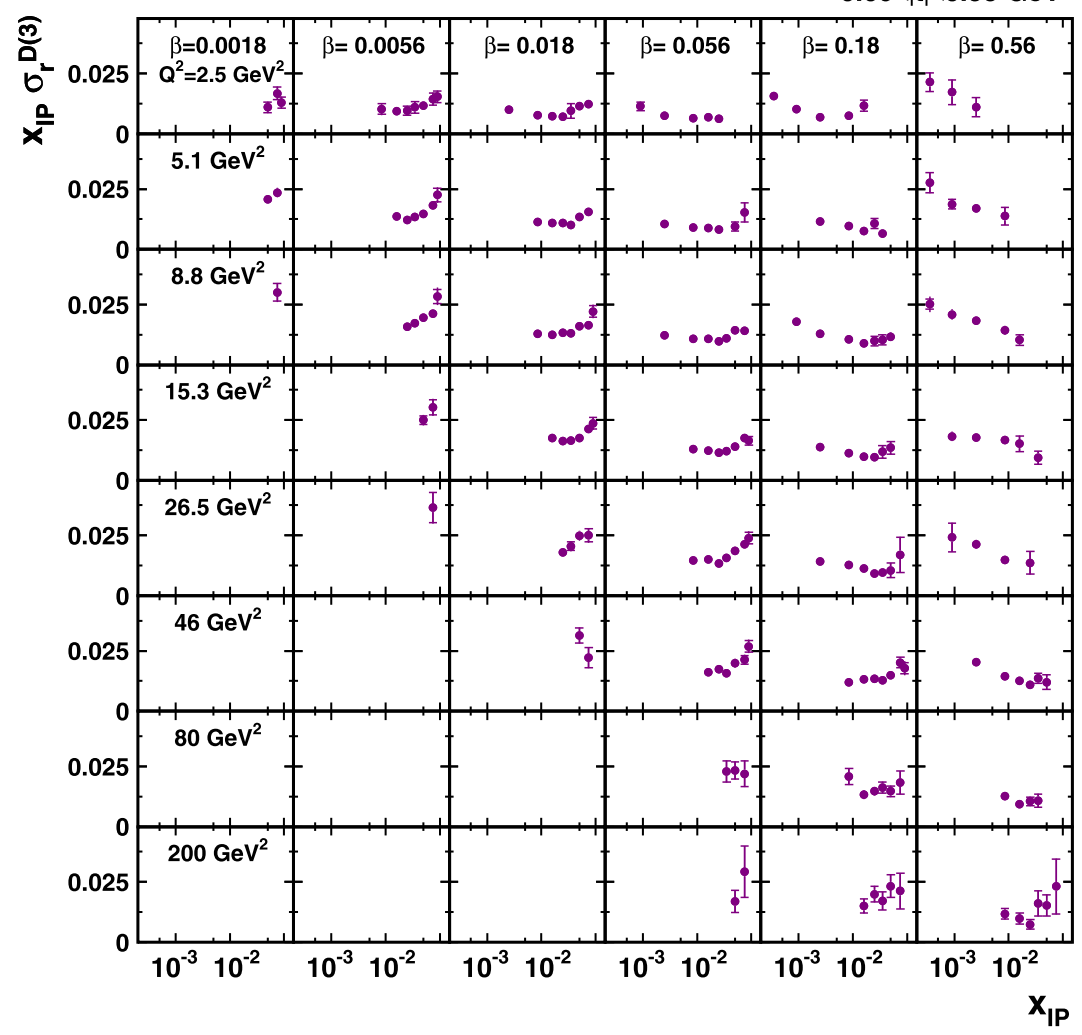


bined. The input data from the two experiments are consistent with a $\chi_{\min }^{2} / n_{\text {dof }}=133 / 161$. The combination of the measurements results in more precise and kinematically extended diffractive DIS data in the $t$-range $0.09<|t|<$ $0.55 \mathrm{GeV}^{2}$. The total uncertainty on the cross section measurement is $6 \%$ for the most precise points. The combined data provide the most precise determination of the absolute normalisation of the $e p \rightarrow e X p$ cross section.

Acknowledgements We are grateful to the HERA machine group whose outstanding efforts have made these experiments possible. We appreciate the contributions to the construction and maintenance of the H1 and ZEUS detectors of many people who are not listed as authors. We thank our funding agencies for financial support, the DESY technical staff for continuous assistance and the DESY directorate for their support and for the hospitality they extended to the non-DESY members of the collaborations.

Open Access This article is distributed under the terms of the Creative Commons Attribution License which permits any use, distribution, and reproduction in any medium, provided the original author(s) and the source are credited.

\section{References}

1. A. Aktas et al. (H1 Collaboration), Eur. Phys. J. C 48, 749 (2006)
2. A. Aktas et al. (H1 Collaboration), Eur. Phys. J. C 71, 1578 (2011)

3. S. Chekanov et al. (ZEUS Collaboration), Eur. Phys. J. C 38, 43 (2004)

4. S. Chekanov et al. (ZEUS Collaboration), Nucl. Phys. B 816, 1 (2009)

5. A. Aktas et al. (H1 Collaboration), Eur. Phys. J. C 48, 715 (2006)

6. F.D. Aaron et al. (H1 Collaboration), Eur. Phys. J. C 72, 2074 (2012)

7. P. Newman, M. Ruspa, arXiv:0903.2957

8. A. Glazov, AIP Conf. Proc. 792, 237 (2005)

9. F.D. Aaron et al. (H1 Collaboration), Eur. Phys. J. C 63, 625 (2009)

10. F.D. Aaron et al. (H1 and ZEUS Collaborations), J. High Energy Phys. 1001, 109 (2010)

11. I. Abt et al. (H1 Collaboration), Nucl. Instrum. Methods Phys. Res. A 386, 310 (1997)

12. I. Abt et al. (H1 Collaboration), Nucl. Instrum. Methods Phys. Res. A 386, 348 (1997)

13. R.D. Appuhn et al. (H1 SPACAL Group), Nucl. Instrum. Methods Phys. Res. A 386, 397 (1997)

14. U. Holm (ed.) (ZEUS Collaboration), The ZEUS detector. Status Report (unpublished), DESY, 1993. Available on http://wwwzeus.desy.de/bluebook/bluebook.html

15. S. Chekanov et al. (ZEUS Collaboration), Nucl. Phys. B 831, 1 (2010) 